\title{
International ERS/ESICM/ESCMID/ALAT guidelines for the management of hospital-acquired pneumonia and ventilator-associated pneumonia
}

Guidelines for the management of hospital-acquired pneumonia (HAP)/ ventilator-associated pneumonia (VAP) of the European Respiratory Society (ERS), European Society of Intensive Care Medicine (ESICM), European Society of Clinical Microbiology and Infectious Diseases (ESCMID) and Asociación Latinoamericana del Tórax (ALAT)

Antoni Torres ${ }^{1,16}$, Michael S. Niederman ${ }^{2,16}$, Jean Chastre ${ }^{3}$, Santiago Ewig ${ }^{4}$, Patricia Fernandez-Vandellos ${ }^{5}$, Hakan Hanberger ${ }^{6}$, Marin Kollef ${ }^{7}$, Gianluigi Li Bassi ${ }^{1}$, Carlos M. Luna ${ }^{8}$, Ignacio Martin-Loeches ${ }^{9}$, J. Artur Paiva ${ }^{10}$, Robert C. Read ${ }^{11}$, David Rigau ${ }^{12}$, Jean François Timsit ${ }^{13}$, Tobias Welte ${ }^{14}$ and Richard Wunderink ${ }^{15}$

@ERSpublications

ERS/ESICM/ESCMID/ALAT evidence-based recommendations for HAP/VAP diagnosis, treatment and prevention http://ow.ly/dGhv30dAVoa

Cite this article as: Torres A, Niederman MS, Chastre J, et al. International ERS/ESICM/ESCMID/ALAT guidelines for the management of hospital-acquired pneumonia and ventilator-associated pneumonia. Eur Respir J 2017; 50: 1700582 [https://doi.org/10.1183/13993003.00582-2017].

ABSTRACT The most recent European guidelines and task force reports on hospital-acquired pneumonia (HAP) and ventilator-associated pneumonia (VAP) were published almost 10 years ago. Since then, further randomised clinical trials of HAP and VAP have been conducted and new information has become available. Studies of epidemiology, diagnosis, empiric treatment, response to treatment, new antibiotics or new forms of antibiotic administration and disease prevention have changed old paradigms. In addition, important differences between approaches in Europe and the USA have become apparent.

The European Respiratory Society launched a project to develop new international guidelines for HAP and VAP. Other European societies, including the European Society of Intensive Care Medicine and the European Society of Clinical Microbiology and Infectious Diseases, were invited to participate and appointed their representatives. The Latin American Thoracic Association was also invited.

A total of 15 experts and two methodologists made up the panel. Three experts from the USA were also invited (Michael S. Niederman, Marin Kollef and Richard Wunderink).

Applying the GRADE (Grading of Recommendations, Assessment, Development and Evaluation) methodology, the panel selected seven PICO (population-intervention-comparison-outcome) questions that generated a series of recommendations for HAP/VAP diagnosis, treatment and prevention.

This article has supplementary material available from erj.ersjournals.com

Received: March 202017 | Accepted after revision: June 132017

This document was endorsed by the ERS Executive Committee, ESCMID and ALAT in July 2017, and by ESICM in August 2017.

Conflict of interest: D. Rigau acts as a methodologist for the European Respiratory Society. All other disclosures can be found alongside this article at erj.ersjournals.com

Copyright @ERS 2017 
Affiliations: ${ }^{1}$ Dept of Pulmonology, Hospital Clínic de Barcelona, Universitat de Barcelona and IDIBAPS, CIBERES, Barcelona, Spain. ${ }^{2}$ Division of Pulmonary and Critical Care Medicine, Weill Cornell Medicine, New York, NY, USA. ${ }^{3}$ Réanimation Médicale, Groupe Hospitalier Pitié-Salpêtrière, Paris, France. ${ }^{4}$ CAPNETZ Stiftung and Thorax Centre in the Ruhr Area, Dept of Respiratory Medicine and Infectious Diseases, Evangelic Hospital in Herne and Augusta Hospital in Bochum, Bochum, Germany. ${ }^{5}$ IDIBAPS, CIBERES, Barcelona, Spain. ${ }^{6}$ Dept of Clinical and Experimental Medicine, Faculty of Medicine and Health Sciences, Linköping University, Linköping, Sweden. ${ }^{7}$ Pulmonary and Critical Care Division, Washington University School of Medicine, St Louis, MO, USA. ${ }^{8}$ Hospital de Clínicas “José de San Martin”, Universidad de Buenos Aires, Ciudad de Buenos Aires, Argentina. ${ }^{9}$ Dept of Clinical Medicine, Wellcome Trust - HRB Clinical Research Facility, St James's Hospital, Trinity College, Dublin, Ireland and CIBERES, Barcelona, Spain. ${ }^{10}$ Emergency and Intensive Care Dept, Centro Hospitalar São João EPE and Dept of Medicine, University of Porto Medical School, Porto, Portugal. "11Academic Unit of Clinical Experimental Sciences and NIHR Southampton Biomedical Research Unit, Faculty of Medicine, and Institute for Life Sciences, University of Southampton, Southampton, UK. ${ }^{12}$ Iberoamerican Cochrane Centre, Barcelona, Spain. ${ }^{13}$ IAME, INSERM UMR 1137, Medical and Infectious Diseases Intensive Care Unit, Paris Diderot University and Bichat Hospital, Paris, France. ${ }^{14}$ Dept of Respiratory Medicine, Medizinische Hoschschule Hannover, Hannover and German Centre of Lung Research (DZL), Germany. ${ }^{15}$ Division of Pulmonary and Critical Care Medicine, Northwestern University Feinberg School of Medicine, Chicago, IL, USA. ${ }^{16}$ These two authors contributed equally to this work.

Correspondence: Antoni Torres, Servei de Pneumologia, Hospital Clinic de Barcelona, Villarroel 170, 08036 Barcelona, Spain. E-mail: atorres@ub.edu

\section{Introduction}

Hospital-acquired pneumonia (HAP) is an infection of the pulmonary parenchyma caused by pathogens that are present in hospital settings [1]. Nosocomial pneumonia develops in patients admitted to the hospital for $>48 \mathrm{~h}$ and usually the incubation period is at least 2 days. Among nosocomial pneumonias, ventilator-associated pneumonia (VAP) develops in intensive care unit (ICU) patients who have been mechanically ventilated for at least $48 \mathrm{~h}$. Patients with severe nosocomial pneumonia who require mechanical ventilation during their treatment after the onset of infection do not meet the definition of VAP. In contrast, ventilator-associated tracheobronchitis (VAT) is characterised by signs of respiratory infection without new radiographic infiltrates, in a patient who has been ventilated for at least $48 \mathrm{~h}$ [2].

HAP is the second most common nosocomial infection and the leading cause of death from nosocomial infections in critically ill patients. Its incidence ranges from 5 to more than 20 cases per 1000 hospital admissions [1], with the highest rates in immunocompromised, surgical and elderly patients. Approximately one-third of nosocomial pneumonia cases, with the majority being VAP, are acquired in the ICU. US epidemiological studies report an incidence of VAP of 2-16 episodes per 1000 ventilator-days $[3,4]$. Cook et al. [5] estimated the risk of VAP to be $3 \%$ per day during the first 5 days on mechanical ventilation, $2 \%$ per day from day 5 to 10 and $1 \%$ per day for the remaining days. However, with respect to earlier reports [6], VAP seems to be on the decrease, probably due to better implementation of preventive strategies. The incidence is still very high (50\%) in trauma and brain injury patients, probably related to the depressed level of consciousness and consequently microaspiration at the time of trauma [7].

HAP and, most prominently, VAP increase duration of hospitalisation and healthcare costs; a recent matched case-control study from a large US database demonstrated longer durations of mechanical ventilation, ICU stay and hospitalisation in patients with VAP than in those without. Worse outcomes have been consistently reported over the years [6-8] and mean hospital charges per VAP patient have increased by approximately USD40000 [7, 8]. In a systematic review of economic analyses of healthcare-associated infections, the mean attributable cost of VAP was USD9969 [9].

In the UK, a conservative estimated cost was GBP10000, which is equivalent to 7 extra days of ICU care; GBP350 was the estimated price of any preventive measure to be considered cost-beneficial [10]. In Turkish University Hospitals, the mean cost of ICU patients with VAP was four times greater compared with those without VAP [11].

Healthcare-associated pneumonia (HCAP) develops in nonhospitalised patients who have multiple risks for being colonised by nosocomial multidrug-resistant (MDR) pathogens [1]. Risk factors for developing HCAP are hospitalisation for $\geqslant 2$ days within the preceding 90 days, residence in a nursing home or

The guidelines published by the European Respiratory Society (ERS) incorporate data obtained from a comprehensive and systematic literature review of the most recent studies available at the time. Health professionals are encouraged to take the guidelines into account in their clinical practice. However, the recommendations issued by this guideline may not be appropriate for use in all situations. It is the individual responsibility of health professionals to consult other sources of relevant information, to make appropriate and accurate decisions in consideration of each patient's health condition and in consultation with that patient and the patient's caregiver where appropriate and/or necessary, and to verify rules and regulations applicable to drugs and devices at the time of prescription. 
extended care facility, home infusion therapy, chronic dialysis, home wound care and contact with subjects colonised by MDR pathogens. Studies in the USA [12] have reported that HCAP is often caused by MDR microorganisms in critically ill patients; in contrast, European data [13] suggest that the aetiology in HCAP patients is similar to that of community-acquired pneumonia (CAP) and that these patients are often not critically ill. For this reason, HCAP management is not covered in these guidelines.

The time of onset of nosocomial pneumonia also affects the possible aetiology, empirical antimicrobial treatment and outcomes [14]. Previously, VAP was categorised as either early- or late-onset VAP [15]. In an interesting study by TrouilLET et al. [16], specific risk factors were strongly associated with infection by MDR pathogens: duration of mechanical ventilation $\geqslant 7$ days (OR 6.0), prior antibiotic use (OR 13.5) and prior use of broad-spectrum drugs (OR 4.1). More recent reports have challenged this classification; indeed, some investigators have found comparable aetiologies in patients with early- or late-onset VAP [17-20]. This may be related to the worldwide rise in MDR pathogens and emphasises that the local ICU ecology is the most important risk factor for acquiring MDR pathogens, irrespective of the length of intubation. The initial HAP or VAP severity (e.g. septic shock) is also a strong risk factor for MDR pathogens, regardless of time of onset.

The crude mortality of nosocomial pneumonia may be as high as 70\% [1]. Several reports have estimated that a third to a half of all VAP-related deaths are the direct result of the infection, with a higher mortality rate in cases caused by Pseudomonas aeruginosa [21] and Acinetobacter spp. [21, 22]. Attributable VAP mortality is defined as the percentage of deaths that would not have occurred in the absence of the infection. Recent studies have reappraised the impact of VAP on mortality [23-25]. In particular, as already mentioned, the risk of VAP is time dependent, and this may cause a significant time-dependent bias because mortality and ICU discharge act as competing end-points. Thus, the most recent studies reported an attributable mortality of $10 \%[25,26]$, with surgical patients and those with mid-range illness severity presenting the highest associated risk

In 2005, the American Thoracic Society (ATS)/Infectious Diseases Society of America (IDSA) published evidence-based guidelines for the management of HAP/VAP [1]. A task force of three European societies (European Respiratory Society (ERS), European Society of Clinical Microbiology and Infectious Diseases (ESCMID) and European Society of Intensive Care Medicine (ESICM)) also published recommendations for HAP and VAP [27]. Since those guidelines were published, a great deal of progress has been made in the understanding of HAP/VAP, e.g. with regard to the different forms of the disease (specifically VAT and VAP), new knowledge about MDR pathogens, new studies for validating guidelines, the bacteriology of HAP in nonintubated patients, new drug development and new trials of aerosolised antibiotics, and new evidence and concepts regarding prevention (e.g. the zero-VAP concept). In addition, regulatory agencies are trying to find surrogate end-points to replace 28-day mortality and to improve the design of randomised clinical trials in this field of investigation. The IDSA and ATS published their latest guidelines in July 2016 [28]. These guidelines differ from the previous recommendations published in 2005 by introducing the use of the GRADE (Grading of Recommendations, Assessment, Development and Evaluation) methodology for evaluation of all available evidence, in removing the concept of HCAP, in using antibiograms to guide antibiotic treatments and in administering short-course therapy for most HAP or VAP patients regardless of their microbial aetiology, as well as antibiotic de-escalation.

The ERS, ESICM, ESCMID and Latin American Thoracic Association (ALAT) all support new evidence-based guidelines for HAP/VAP, and have appointed a panel of experts to develop clinical recommendations. Although the IDSA/ATS were also developing new guidelines, the panel thought that a European perspective was needed in view of the differences between the US and European approaches in several areas:

1) The use of ventilator-associated complications [29] as a surrogate measure of VAP has become very popular in the USA in recent years for benchmarking purposes. However, because of its lack of sensitivity and specificity it has not been widely implemented in Europe.

2) There are differences in the definitions of HAP and VAP.

3) Diagnosis of HAP/VAP is still a matter of controversy, particularly with regard to the role of quantitative cultures and bronchoscopic sampling. Different approaches are applied in Europe and the USA $[1,28]$.

4) The efficacy of certain antibiotics varies widely in different geographic regions, as does the frequency of MDR pathogens in different European countries [17].

5) Attitudes and beliefs about how to best prevent pneumonia, including the use of selective digestive decontamination (SDD), differ considerably. This is due mainly to the wide variation in VAP incidence between Europe and the USA $[28,29]$. In particular, following an extensive implementation of ventilator bundles in the last decade in the USA there has been a consistent drop in VAP rates. 
This is not the case in Europe, where incidence remains high in many ICUs in spite of the consistent use of ventilator bundles [30].

6) Antimicrobial stewardship is an important issue in both Europe and the USA, but the approach to this problem in the two continents differs considerably, particularly in relation to the need for prior authorisation of certain antibiotics before their use in the ICU as part of empiric therapy [31]. In addition, in some European countries there is such a strong emphasis on stewardship that physicians may be reluctant to use broad-spectrum empiric therapy that is intended to target at least $95 \%$ of the likely pathogens, an implied goal of the latest IDSA/ATS guidelines.

\section{Scope and purpose}

The purpose of this document is to provide guidance on the most effective treatments and management strategies for adult patients with HAP and VAP. The recommendations reported in these guidelines may not apply to patients with a secondary immune deficiency (related to HIV infection, treatment or disease-induced immunosuppression) or primary immune deficiency; in these patients, HAP and VAP can be caused by a broad spectrum of microorganisms, and the diagnostic and therapeutic approaches are very different.

These guidelines are intended mainly for specialists in respiratory medicine and critical care managing adults with HAP or VAP. They may also be of interest to general internists, specialists in infectious diseases, pharmacists, microbiologists and policy makers.

\section{Methods}

These guidelines were developed by a committee of experts from the ERS, ESICM, ESCMID and ALAT. The committee included specialists in respiratory medicine with expertise in the management of patients with lung infections, intensive care specialists, as well as microbiologists and methodologists with experience in evidence synthesis and guideline development.

The committee's first face-to-face meeting was held in February 2013, where a total of seven clinical questions were formulated. The guideline process continued with a series of telephone conferences and electronic-based discussions between committee members. A second face-to-face meeting was held in Barcelona (February 2015) to decide on the guideline recommendations.

In collaboration with the methodologists, a search strategy was designed using key terms and keywords for each clinical question. The search was limited to human studies (systematic reviews, randomised clinical trials or observational studies) written in English. The PubMed platform was used to search MEDLINE. The Cochrane Central Register of Controlled Trials (CENTRAL), the Cochrane Database of Systematic Reviews and the National Health Service's Economic Evaluation Database were also searched to find additional studies and economic evaluations. All searches were performed until December 2014 and guideline panel members monitored the literature relevant to their assigned clinical question up to September 2016. The search retrieved 5560 citations; after the review of titles and abstracts, and full-text when needed, a total of 109 references were included for analysis (figure 1).

\section{Assessment of the level of evidence and grade of recommendations}

Evidence levels and recommendation grades used in these guidelines follows the GRADE methodology $[32,33]$. GRADE has four levels of evidence: high, moderate, low and very low. Recommendations are classified as strong or weak after considering the quality of the evidence, the balance of desirable and undesirable consequences of the management options compared, the assumptions about the relative importance of outcomes, the implications for resource use, and the acceptability and feasibility of implementation. Recommendations and their strength were decided by consensus and, if required, by voting. Supplementary table S1 provides a suggested interpretation of these recommendations by the targeted stakeholders, who include patients, clinicians and health policy makers.

These guidelines will be considered for revision in 2020, or sooner if relevant new evidence becomes available.

\section{PICO questions and recommendations}

All PICO (population-intervention-comparison-outcome) questions and corresponding recommendations are listed in table 1. 


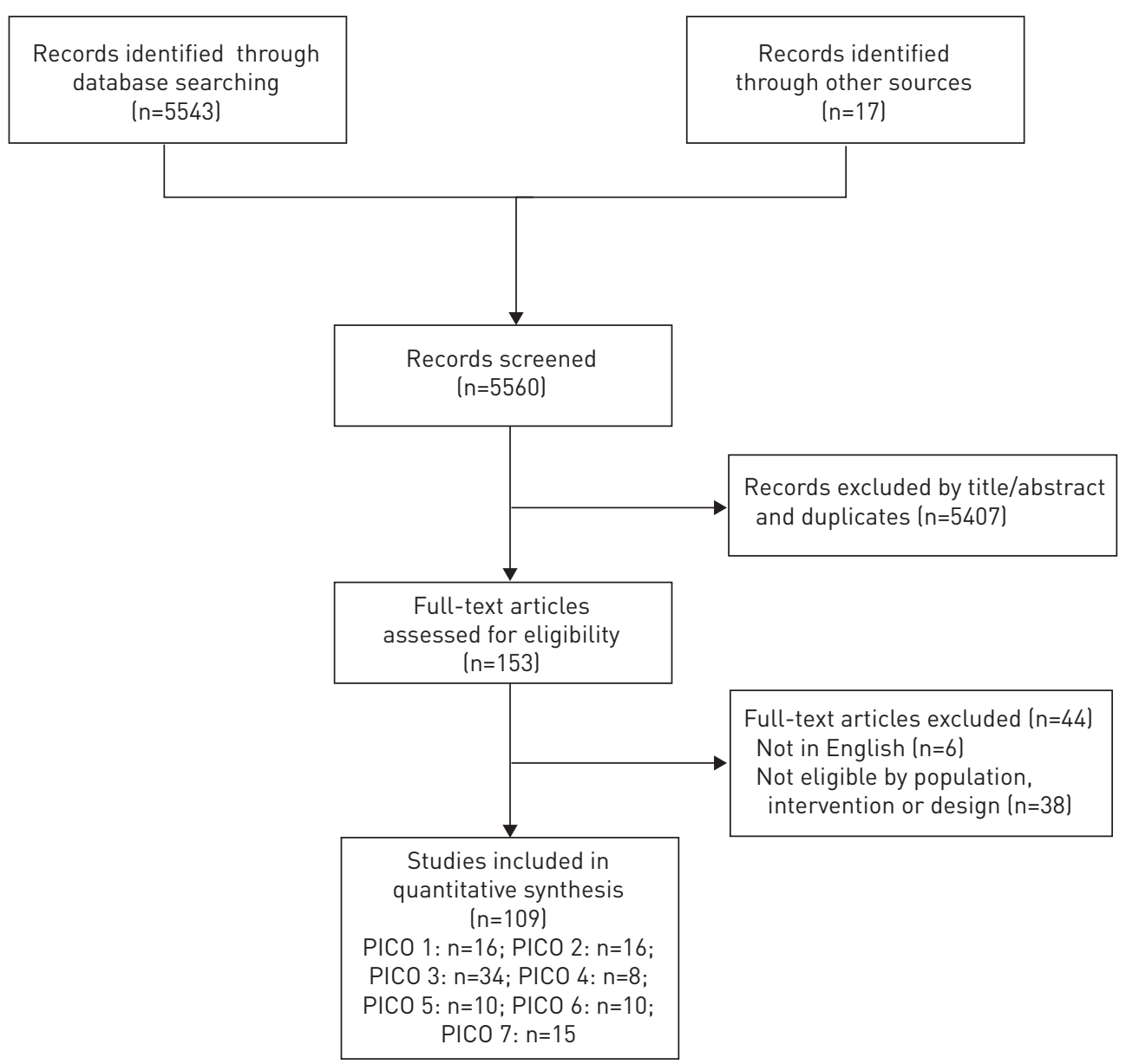

FIGURE 1 Literature search.

Question 1: In intubated patients suspected of having VAP, should distal quantitative samples be obtained instead of proximal quantitative samples?

Recommendations

We suggest obtaining distal quantitative samples (prior to any antibiotic treatment) in order to reduce antibiotic exposure in stable patients with suspected VAP and to improve the accuracy of the results. (Weak recommendation, low quality of evidence.)

We recommend obtaining a lower respiratory tract sample (distal quantitative or proximal quantitative or qualitative culture) to focus and narrow the initial empiric antibiotic therapy. (Strong recommendation, low quality of evidence.)

\section{Benefits and harms}

Invasive techniques require the participation of qualified clinicians, may compromise gas exchange during the procedure and may be associated with higher direct costs [34-37]. However, a pooled analysis of five randomised controlled trials (RCTs) did not show any difference in overall mortality between VAP patients diagnosed through invasive or noninvasive techniques (profile 2 in the supplementary material) [34]. No RCTs have compared qualitative and quantitative cultures of the same bacteriological sample. Quantitative cultures help to guide initial antibiotic therapy for VAP; when available, they allow the precise identification of the causative organisms and susceptibility patterns, thus providing invaluable information for optimal antibiotic selection. However, antibiotic therapy for a current episode of HAP/VAP can alter and modify the results from quantitative cultures when samples are obtained within $48 \mathrm{~h}$ of starting a new antibiotic regimen.

Three RCTs have compared the effectiveness of invasive methods using quantitative cultures versus noninvasive methods using qualitative cultures [34, 35, 37]. A pooled analysis did not show any significant influence of the approach used on the change of the initial antibiotic regimen [34]. The studies were not 
TABLE 1 PICO questions and recommendations

Question 1: In intubated patients suspected of having VAP, should distal quantitative samples be obtained instead of proximal quantitative samples?
Question 2: Can patients suspected of having nosocomial pneumonia (HAP and VAP), who have early-onset infection and none of the classic risk factors for MDR pathogens, be treated appropriately if they receive a different and narrower spectrum empiric therapy than patients with late-onset infection and/or the presence of MDR risk factors?
Question 3: When using initial broad-spectrum empiric therapy for HAP/VAP, should it always be with two drugs or can it be with one drug and, if starting with two drugs, do both need to be continued after cultures are available?
We suggest obtaining distal quantitative samples (prior to any antibiotic treatment) in order to reduce antibiotic exposure in stable patients with suspected VAP and to improve the accuracy of the results. (Weak recommendation, low quality of evidence.)

We recommend obtaining a lower respiratory tract sample (distal quantitative or proximal quantitative or qualitative culture) to focus and narrow the initial empiric antibiotic therapy. (Strong recommendation, low quality of evidence.)

We suggest using narrow-spectrum antibiotics lertapenem, ceftriaxone, cefotaxime, moxifloxacin or levofloxacin) in patients with suspected low risk of resistance and early-onset HAP/VAP. (Weak recommendation, very low quality of evidence.)

Remarks: The risk of Clostridium difficile infections is increased with third-generation cephalosporins compared with penicillins or quinolones. The panel found it reasonable to consider as "low risk" patients without septic shock, with no other risk factors for MDR pathogens and those who are not in hospitals with a high background rate of resistant pathogens. However, the presence of other clinical conditions may make individuals unsuitable for this recommendation. The rate of resistant pathogens is highly variable across different countries, settings and hospitals. A prevalence of resistant pathogens in local microbiological data $>25 \%$ is considered a high background rate (the rate of resistance in the ICU caring for the patient (not the hospital as a whole) is the relevant factor to consider).

We recommend broad-spectrum empiric antibiotic therapy targeting Pseudomonas aeruginosa and extended-spectrum $\beta$-lactamaseproducing organisms, and, in settings with a high prevalence of Acinetobacter spp., in patients with suspected early-onset HAP/VAP who are in septic shock, in patients who are in hospitals with a high background rate of resistant pathogens present in local microbiological data and in patients with other (nonclassic) risk factors for MDR pathogens (see Question 3). (Strong recommendation, low quality of evidence.)

The panel believes that tailoring antibiotic therapy to the susceptibility data of the aetiological pathogen once microbiological and clinical response data become available (day 3 ) represents good practice. (Good practice statement.)

We recommend initial empiric combination therapy for high-risk HAP/ VAP patients to cover Gram-negative bacteria and include antibiotic coverage for MRSA in those patients at risk. (Strong recommendation, moderate quality of evidence.)

Remarks: The panel finds it reasonable to consider as "high-risk HAP/VAP" patients who present HAPNAP and either septic shock and/or the following risk factors for potentially resistant microorganisms: hospital settings with high rates of MDR pathogens (i.e. a pathogen not susceptible to at least one agent from three or more classes of antibiotics), previous antibiotic use, recent prolonged hospital stay ( $>5$ days of hospitalisation) and previous colonisation with MDR pathogens. The rate of resistant pathogens varies widely across different countries, settings and hospitals. However, a prevalence of resistant pathogens in local microbiological data $>25 \%$ represents a high-risk situation (including Gram-negative bacteria and MRSA).

If initial combination therapy is started, we suggest continuing with a single agent based on culture results and only consider maintaining definitive combination treatment based on sensitivities in patients with extensively drug-resistant (XDR; i.e. susceptible to only one or two classes of antibiotics)/pan-drug-resistant (PDR; i.e. not susceptible to any antibiotics) nonfermenting Gram-negative bacteria and carbapenem-resistant Enterobacteriaceae (CRE) isolates. (Weak recommendation, low quality of evidence.) 
Remarks: The panel finds it reasonable to consider selected patients at low risk for MDR pathogens (see Question 2) and some patients at high risk for MDR pathogens for initial empiric monotherapy, if there is a single-antibiotic therapy that is effective against $>90 \%$ of Gram-negative bacteria according to the local antibiogram. However, other clinical conditions, particularly severe illness or septic shock, may make individuals unsuitable for this recommendation.

Question 4: In patients with HAP/VAP, can duration of antimicrobial therapy be shortened to 7-10 days for certain populations, compared with 14 days, without increasing rates of relapsing infections or decreasing clinical cure?
Question 5: In patients receiving antibiotic treatment for VAP or $H A P$, is bedside clinical assessment equivalent to the detection of serial biomarkers to predict adverse outcomes/clinical response at 72-96 $\mathrm{h}$ ?
Question 6: In patients with HAP with severe sepsis or VAP, can serum PCT be used to reduce the duration of antibiotic therapy, compared with care that is not guided by serial biomarker measurements?
We suggest using a 7-8-day course of antibiotic therapy in patients with VAP without immunodeficiency, cystic fibrosis, empyema, lung abscess, cavitation or necrotising pneumonia and with a good clinical response to therapy. (Weak recommendation, moderate quality of evidence.)

Remarks: This recommendation also includes patients with nonfermenting Gram-negatives, Acinetobacter spp. and MRSA with a good clinical response. Longer courses of antibiotics may be needed in patients with inappropriate initial empiric therapy, and should be individualised to the patient's clinical response, specific bacteriological findings /such as PDR pathogens, MRSA or bacteraemial and the serial measurement of biomarkers when indicated (see Question 6 and table 3).

The panel believes that applying the rationale and recommendations used for VAP in nonventilated patients with HAP represents good practice. (Good practice statement.)

We suggest against routine treatment with antibiotics for $>3$ days in patients with low probability of HAP and no clinical deterioration within $72 \mathrm{~h}$ of symptom onset. (Weak recommendation, low quality of evidence.)

Remarks: The term "low probability of HAP" refers to patients with low Clinical Pulmonary Infection Score (CPIS) scores or a clinical presentation not highly suggestive of pneumonia (e.g. $\leqslant 6$ ) at symptom onset and continuing up to $72 \mathrm{~h}$.

The panel believes that performing routine bedside clinical assessment in patients receiving antibiotic treatment for VAP or HAP represents good practice. (Good practice statement.)

Remarks: Clinical evaluation usually involves measurement of temperature, tracheobronchial secretion volume, culture and purulence assessment of tracheobronchial secretions, evaluation for chest radiograph resolution, white blood cell count, arterial oxygen tension/inspiratory oxygen fraction $\left(\mathrm{PaO}_{2} / \mathrm{FIO}_{2}\right)$, and calculation of one or more scores such as CPIS, ODIN (Organ Dysfunction and Infection System), SOFA (Sequential Organ Failure Assessment), SAPS II (Simplified Acute Physiological Score II) and APACHE II.

We do not recommend routinely performing biomarker determinations in addition to bedside clinical assessment in patients receiving antibiotic treatment for VAP or HAP to predict adverse outcomes and clinical response at 72-96 h. (Strong recommendation, moderate quality of evidence.)

Remarks: Biomarker determinations may include $C$-reactive protein (CRP), procalcitonin (PCT), copeptin and mid-regional pro-atrial natriuretic peptide (MR-proANP). Clinicians should take into consideration the availability, feasibility and costs of each biomarker before routine testing.

We do not recommend the routine measurement of serial serum PCT levels to reduce duration of the antibiotic course in patients with HAP or VAP when the anticipated duration is 7-8 days. (Strong recommendation, moderate quality of evidence.)

The panel believes that the measurement of serial serum PCT levels together with clinical assessment in specific clinical circumstances (see table 3) with the aim of reducing antibiotic treatment duration represents good practice. (Good practice statement.) 
TABLE 1 Continued

PICO question

Question 7: In patients requiring mechanical ventilation for $>\mathbf{4 8} \mathrm{h}$, does topical application of nonabsorbable antimicrobials (antibiotics or chlorhexidine) in the oropharynx (SOD) or in the oropharynx and intestinal tract along with intravenous antibiotics (SDD) reduce the risk of VAP occurrence and/or improve patient outcome compared with standard care? (Standard care being treatment dispensed in the ICU by the medical team in their usual manner]

\section{Recommendations}

The guideline panel decided not to issue a recommendation on the use of chlorhexidine to perform selective oral decontamination (SOD) in patients requiring mechanical ventilation until more safety data become available, due to the unclear balance between a potential reduction in pneumonia rate and a potential increase in mortality. (No formal recommendation.)

We suggest the use of SOD, but not SDD, in settings with low rates of antibiotic-resistant bacteria and low antibiotic consumption (where low antibiotic consumption in the ICU is $<1000$ daily doses per 1000 admission days). (Weak recommendation, low quality of evidence.)

Remarks: Although establishing a cut-off value for low and high resistance settings is a dilemma, the committee felt that a $5 \%$ threshold was reasonable.

VAP: ventilator-associated pneumonia; HAP: hospital-acquired pneumonia; MDR: multidrug-resistant; ICU: intensive care unit; MRSA: methicillin-resistant Staphylococcus aureus; APACHE II: Acute Physiology and Chronic Health Evaluation II; SDD: selective digestive decontamination.

blinded and the results varied widely. The fact that some investigators may be reluctant to withhold therapy until quantitative results are available may explain these findings.

The number of antibiotic-free days was assessed in two RCTs, although the results were not pooled [34, 35]. In one study with 413 patients, the invasive distal quantitative strategy was combined with an algorithm for treatment de-escalation and led to a significant increase in antibiotic-free days at day 14 (5.0 \pm 5.1 versus $2.2 \pm 3.5)$ and day 28 (11.5 \pm 9.0 versus $7.5 \pm 7.6)$ compared with noninvasive methods using qualitative cultures. The difference was statistically significant for all antibiotic classes except carbapenems [37]. In that study, all microorganisms recovered from qualitative culture, including potentially nonpathogenic organisms such as coagulase-negative staphylococci, were treated and antibiotics may have been overused in the qualitative proximal culture arm [37]. In the Canadian Critical Care Trial Group study, antibiotic-free days at day 28 were similar between groups [34]. In that study, there were no clear recommendations on antimicrobial de-escalation and the research protocol may have facilitated appropriate discontinuation of antibiotics or targeted therapy in the two groups, thus minimising the differences between them [34].

Overall mortality, ICU length of stay and duration of mechanical ventilation did not show differences between the two interventions in a pooled analysis [34]. The potential downsides of narrowing antimicrobial therapy in response to the results of quantitative cultures have also been evaluated in cohort studies, in which this diagnostic strategy was not associated with an increase in mortality or length of stay (profile 1 in the supplementary material) $[37,38]$.

Noninvasive diagnostic methods (e.g. endotracheal aspirate collection) led to an over-identification of bacteria by initial direct examination of samples. In one clinical trial, bacterial identification was achieved by endotracheal qualitative aspirates in $86 \%$ of the patients, but in only $43 \%$ with the use of bronchoscopic distal quantitative methods [35]. This important difference in bacterial identification may explain the reduction in antibiotic-free days and overall antibiotic exposure between the two approaches seen in previous trials. The link between antibiotic use and antibiotic resistance, both inside a unit and at an individual level, on the infecting flora and on the gut microbiota has been clearly identified [39]. Moreover, a recent observational study in 89 patients with clinically suspected VAP and a negative $\left(<10^{4} \mathrm{CFU} \cdot \mathrm{mL}^{-1}\right)$ quantitative bronchoalveolar lavage (BAL) compared patients with early (within 1 day) and late antibiotic discontinuation. Despite similar severity scores, there were no differences in mortality between patients with early $(25.0 \%)$ and late $(30.6 \%)$ discontinuation. There were significantly fewer superinfections $(22.5 \%$ versus $43 \%)$, respiratory superinfections (10.\% versus $29 . \%)$ and $\mathrm{MDR}$ superinfections $(7.5 \%$ versus $36 \%$ ) in the early than in the late discontinuation group [40].

Nevertheless, the collection of a bacteriological sample before any change in antimicrobial therapy allows the immediate withdrawal of the antibiotic following a negative finding and a subsequent de-escalation according to the microorganisms grown from bacteriological culture [35, 37, 41, 42]. This may not be feasible in all situations. In practice, when antimicrobials have recently been modified, both qualitative and quantitative samples lose their sensitivity and specificity [43-47]. A negative finding indicates either that the patient has been successfully treated for pneumonia and that the bacteria were eradicated (but 
de-escalation may not always be possible) or that the lung infection was not present to begin with (leading to an active search for other diseases and withdrawal or adjustment of antimicrobial therapy). To cope with this problem, if bacteriological analyses are not available immediately, processing of a bacteriological specimen collected after refrigeration can offer good reliability [48].

The guideline panel noted that invasive techniques using quantitative cultures are widely available and feasible at most of the specialised centres that care for patients with VAP. Panel members felt that the overall benefits in terms of antibiotic exposure probably outweigh the harms in comparison with noninvasive methods using qualitative cultures, particularly if samples are collected before new antibiotics are started. In critically ill VAP patients, the benefits of invasive techniques are less clear, due to the potential deleterious impact of bronchoscopy on gas exchange, especially in patients with severe acute respiratory distress syndrome and profound (unstable) septic shock. Mini-BAL can partially overcome these deleterious effects.

\section{Relative importance of the outcomes}

The panel placed greater value on the potential benefits of reducing antibiotic exposure (and its impact on antibiotic resistance) than on the potential complications of invasive techniques. However, there is some concern that if the procedure is performed shortly after a recent change in antibiotics or at a centre without technical expertise, a false-negative result may mean that patients are not treated in an efficient and timely manner.

\section{Resource use}

No appropriate cost-effectiveness studies have been identified. The panel took into consideration the potentially high costs due to the future of emerging antibiotic resistance with the routine use of broad-spectrum, and prolonged, courses of antibiotics and the reduced direct costs related to a short course of antibiotics.

Question 2: Can patients suspected of having nosocomial pneumonia (HAP and VAP), who have early-onset infection and none of the classic risk factors for MDR pathogens, be treated appropriately if they receive a different and narrower spectrum empiric therapy than patients with late-onset infection and/or the presence of MDR risk factors?

\section{Recommendations}

We suggest using narrow-spectrum antibiotics (ertapenem, ceftriaxone, cefotaxime, moxifloxacin or levofloxacin) in patients with suspected low risk of resistance and early-onset HAP/VAP. (Weak recommendation, very low quality of evidence.)

Remarks: The risk of Clostridium difficile infections is increased with third-generation cephalosporins compared with penicillins or quinolones. The panel found it reasonable to consider as "low risk" patients without septic shock, with no other risk factors for MDR pathogens and those who are not in hospitals with a high background rate of resistant pathogens. However, the presence of other clinical conditions may make individuals unsuitable for this recommendation. The rate of resistant pathogens is highly variable across different countries, settings and hospitals. A prevalence of resistant pathogens in local microbiological data $>25 \%$ is considered a high background rate (the rate of resistance in the ICU caring for the patient (not the hospital as a whole) is the relevant factor to consider).

We recommend broad-spectrum empiric antibiotic therapy targeting $P$. aeruginosa and extended-spectrum $\beta$-lactamase (ESBL)-producing organisms, and, in settings with a high prevalence of Acinetobacter spp., in patients with suspected early-onset HAP/VAP who are in septic shock, in patients who are in hospitals with a high background rate of resistant pathogens present in local microbiological data and in patients with other (nonclassic) risk factors for MDR pathogens (see Question 3). (Strong recommendation, low quality of evidence.)

The panel believes that tailoring antibiotic therapy to the susceptibility data of the aetiological pathogen once microbiological and clinical response data become available (day 3) represents good practice. (Good practice statement.)

\section{Benefits and harms}

The efforts to define a population of nosocomial pneumonia patients who can receive appropriate narrow-spectrum empiric antibiotic therapy rather than broad-spectrum multidrug therapy may help to prevent the overuse of our most effective antibiotics and thus avoid future resistance. In addition, the use of a focused, narrower spectrum regimen may forestall some of the side-effects associated with the use of multiple, broad-spectrum antibiotics. 
Our search did not find any RCTs comparing the effectiveness of broad- and narrow-spectrum empiric antibiotic use in patients with anticipated low-risk MDR pathogens. Available RCTs comparing mono versus dual empirical antibiotic therapy in patients with VAP/HAP have specifically excluded patients with high disease severity. Even in this population, all patients received broad-spectrum antibiotics.

Time of onset of pneumonia has been extensively described in the literature as an important risk factor for specific pathogens, mainly MDR pathogens. Early-onset HAP and VAP, defined as occurring within the first 4 days of hospitalisation, usually carry a better prognosis and are more likely to be caused by antibiotic-sensitive bacteria than other types of pneumonia. Late-onset HAP and VAP ( $\geqslant 5$ days of hospitalisation) are more likely to be caused by MDR pathogens, and are associated with increased patient mortality and morbidity [17]. However, even with early-onset HAP/VAP, the presence of other classic risk factors for resistance narrows the population that might potentially benefit from less broad-spectrum therapy $[1,18]$. These risk factors have been identified as previous antimicrobial therapy or hospitalisation $(\geqslant 2$ days) in the preceding 90 days and, more recently, the nonclassic risk of having a high frequency of antibiotic resistance in the community or in the specific hospital unit $[1,18]$.

Several studies showed that the percentage of MDR pathogens among patients with early-onset VAP varied from as low as $10 \%$ to as high as $51 \%$. In general, if the overall incidence of MDR pathogen VAP is $>25 \%$, the frequency of MDR pathogens in early-onset pneumonia is similar to the overall frequency of MDR pathogens causing nosocomial pneumonia (table 2 and profile 3 in the supplementary material).

One prospective observational cohort study of 689 mechanically ventilated patients with nosocomial pneumonia showed that 152 out of 485 patients with a confirmed microbiological diagnosis had early-onset ( $<5$ days of mechanical ventilation) pneumonia with no classic risk factors for MDR pathogens. 77 out of these 152 patients (51\%) were infected with potentially resistant microorganisms, and these organisms were associated with the presence of severe sepsis or septic shock (OR 3.7) and the development of pneumonia in centres with a prevalence of MDR pathogens $>25 \%$ (OR 11.3) (profile 3 in the supplementary material) [18].

One further prospective observational study in 276 patients with ICU-acquired pneumonia (146 with VAP) classified patients into early onset without risk factors for MDR pathogens (38 patients) and late onset or with risk factors for MDR pathogens (238 patients) according to the 2005 ATS/IDSA guidelines $[1,19]$. The incidence of MDR pathogens did not differ between the two groups (26\% and 29\%, respectively). However, there were some patients with early-onset pneumonia who did not have classic risk factors for MDR pathogens; $46 \%$ had current or former alcohol abuse, 37\% had recent surgery, $34 \%$ had chronic heart disease, $24 \%$ had chronic lung disease, $24 \%$ had diabetes mellitus and $18 \%$ had previous use of corticosteroids. Only $18 \%$ of the patients with early onset and no risk factors for MDR pathogens underwent therapy that complied with the recommendations of the 2005 ATS/IDSA guidelines regarding the use of limited-spectrum antibiotic therapy and from those $43 \%$ presented initial nonresponse to therapy (profile 4 in the supplementary material) [19].

In another prospective cohort study assessing the risk factors for the isolation of pathogens that are potentially resistant to multiple drugs in ICU-acquired pneumonia, the strongest predictors for infection with MDR pathogens were older age and prior antibiotics either as prophylaxis (OR 4.6) or as therapy (OR 8.2). In that study, an early-onset HAP was defined as occurring $<5$ days after admission and $52 \%$ of this population had MDR pathogens. The risk of infection with either P. aeruginosa or ESBL-producing

TABLE 2 Relationship between the frequency of multidrug-resistant (MDR) pathogens in early-onset nosocomial pneumonia (EOP) ${ }^{\#}$ versus the overall frequency of MDR pathogens causing hospital-acquired pneumonia (HAP)

\begin{tabular}{|c|c|c|}
\hline First author [ref.] & MDR in EOP \% & MDR in HAP overall \% \\
\hline MontRaVers [49] & Similar to overall & 34 \\
\hline LEROY [50] & 19 & 30 \\
\hline FERRER [19] & & 26 \\
\hline Perbet [51] & & Similar to overall \\
\hline Restrepo [20] & 27.8 & 30 \\
\hline Martin-Loeches [18] & 51 & 57 \\
\hline ARVANitis [52] & 10 & 25 \\
\hline Verhamme [53] & 52 & \\
\hline
\end{tabular}

${ }^{\#}$ : EOP was defined as occurring $\leqslant 5$ days after admission. 
organisms rose in parallel with time in the ICU, occurring in only $10 \%$ of infections that began $<4$ days after admission, but in 34\% of infections beginning between days 6 and 9 after admission (profile 3 in the supplementary material) [53].

In an observational prospective cohort study including 124 patients with bacteriologically confirmed HAP and an overall incidence of MDR pathogens of 30\%, the multivariate analysis identified certain factors associated with a lower risk of MDR pathogens. The combination of these factors in a cohort of 26 patients allowed the validation of an algorithm that identified all patients with antimicrobial-susceptible HAP. The absence of prior antimicrobial treatment, the presence of prior antimicrobial treatment with neurological disturbances on ICU admission and early-onset pneumonia, and the presence of prior antimicrobial treatment without neurological disturbances but with aspiration on ICU admission were always associated with antimicrobial-susceptible HAP [50].

The major concern when using empiric narrow-spectrum therapy, even in selected patients, is that not all the aetiological pathogens will be treated if the patient is actually infected with an MDR pathogen. A prospective observational study conducted to define the impact of BAL data on the selection of antibiotics and the outcomes of patients with VAP concluded that when adequate antibiotic therapy was initiated early in patients with a strong clinical suspicion of VAP, the mortality rate (38\%) was lower in comparison with inadequate therapy (91\%) or no therapy (60\%). Even when patients were switched to an adequate therapy when BAL data became available, mortality was comparable to those who continued to receive inadequate therapy [54].

In addition, a prospective cohort study carried out to assess the rate of appropriateness of empirical antimicrobial therapy in 115 VAP patients showed that the mortality rate was significantly higher in the patients with inappropriate empirical therapy than in those with appropriate treatment (47\% and $20 \%$, respectively). A limited-spectrum therapy was used in 79 patients (69\%) according to the criteria of early-onset VAP ( $<5$ days) without recent prior hospitalisation or prior antibiotic treatment. Treatment was escalated in 21 out of 79 patients (27.\%) by either adding another antibiotic, using broader-spectrum therapy or both (profile 4 in the supplementary material) [55].

A group of observational retrospective or cohort studies including mixed populations has suggested the presence of other additional factors related to either an increase or a decrease of MDR pathogen incidence. Age $>65$ years was associated with a higher risk of methicillin-resistant Staphylococcus aureus (MRSA) infection [52]; gastric acid suppressive therapy, tube feeding, chronic dialysis and congestive heart failure may increase the incidence of MDR pathogens in either HAP or CAP [56]. Surgery can be a surrogate marker of prior antibiotic therapy as a prophylaxis which was associated with a high incidence of Gram-negative bacteria or staphylococcal early-onset pneumonias [49]. Acute renal failure was associated with a higher risk of CAP due to P. aeruginosa, ESBL-producing Enterobacteriaceae and MRSA [57]. Early aspiration in patients who have been resuscitated from cardiac arrest has been associated with a relatively low frequency of MDR pathogens [51].

\section{Relative importance of the outcomes}

The direct consequences of narrow- or broad-spectrum empiric antibiotic therapy in patients with a low probability of MDR pathogens have not been assessed. The guideline panel considered that the appropriateness of treatment is an adequate surrogate outcome for the important direct consequences of empiric treatment. Due to the large number of patients at risk for MDR pathogens, the guideline panel placed higher value on appropriateness of treatment than on the emergence of resistance or adverse events.

\section{Resource use}

There are no cost-effectiveness studies comparing the use of using narrow- or broad-spectrum empiric therapy in HAP or VAP.

Use of narrow-spectrum therapy may be associated with lower direct costs due to reduced drug acquisition and drug-related toxicity costs, and may potentially reduce the emergence of MDR pathogens, which in turn are very costly to contain and manage. However, it still remains to be determined whether the use of a narrow-spectrum agent in appropriate patients will lead to a cost benefit.

In contrast, if narrow-spectrum empiric antibiotic therapy leads to inappropriate therapy, it may be associated with higher costs due to prolonged mechanical ventilation and length of stay.

Some data point to a higher utilisation of resources when MDR pathogens are present. In a large European study, HAP patients with potentially resistant microorganisms had fewer ventilator-free days, longer ICU stay, longer hospital stay and more use of combination antibiotic therapy [17]. Similarly, a 
study of 200 VAP patients found that patients with MDR infection had longer ICU stay and mechanical ventilation than patients without these pathogens [52].

\section{Balance between desirable effects and undesirable effects}

The percentage of potentially MDR pathogens is significant even in early-onset HAP/VAP and several studies have also identified risk factors for MDR pathogens in patients with either nosocomial or HCAP, regardless of the time of infection onset [20]. As a consequence, if certain factors (other than the classic MDR risk factors) are present, this might lead to the presence of MDR pathogens in a patient with early-onset VAP and the use of narrow-spectrum therapy would not be an appropriate choice.

At the present time, the number of patients with early-onset HAP who can safely receive empiric narrow-spectrum therapy is limited. Selection should be based on an assessment of individual risk factors, severity of illness and the local frequency of MDR pathogens in the ICU in question. Inappropriate therapy may increase nonresponse to therapy, which can in turn prolong the duration of mechanical ventilation, antibiotic use and ICU length of stay, and may also increase inpatient mortality.

Even though there are doubts about the effectiveness of using broad-spectrum empiric antibiotic therapies in most patients with suspected HAP/VAP, the guideline panel considered that the potential benefits outweigh the risks.

If the use of a narrow-spectrum agent as empiric therapy is possible in a selected group of patients, this may reduce the emergence of antibiotic-resistant organisms in intubated ICU patients, as antibiotic use is known to promote subsequent resistance in both individual patients and in the ICU in general. In addition, avoidance of multiple, broad-spectrum drugs may prevent drug-related toxicities, including drug-induced renal insufficiency (e.g. with the use of nephrotoxic agents such as aminoglycosides and vancomycin) [58].

In the future, as rapid diagnostic tests (particularly PCR) become available [59], it may be possible to rely on the high sensitivity (i.e. the ability to detect both colonising and infecting pathogens) of these methods and to select a narrow-spectrum empiric therapy if no MDR pathogens are identified from a deep respiratory tract sample (endotracheal aspirate or BAL).

Question 3: When using initial broad-spectrum empiric therapy for HAP/VAP, should it always be with two drugs or can it be with one drug and, if starting with two drugs, do both need to be continued after cultures are available?

Recommendations

We recommend initial empiric combination therapy for high-risk HAP/VAP patients to cover Gram-negative bacteria and include antibiotic coverage for MRSA in those patients at risk. (Strong recommendation, moderate quality of evidence.)

Remarks: The panel finds it reasonable to consider as "high-risk HAP/VAP" patients who present HAP/VAP and either septic shock and/or the following risk factors for potentially resistant microorganisms: hospital settings with high rates of MDR pathogens (i.e. a pathogen not susceptible to at least one agent from three or more classes of antibiotics), previous antibiotic use, recent prolonged hospital stay ( $>5$ days of hospitalisation) and previous colonisation with MDR pathogens. The rate of resistant pathogens varies widely across different countries, settings and hospitals. However, a prevalence of resistant pathogens in local microbiological data $>25 \%$ represents a high-risk situation (including Gram-negative bacteria and MRSA).

If initial combination therapy is started, we suggest continuing with a single agent based on culture results and only consider maintaining definitive combination treatment based on sensitivities in patients with extensively drug-resistant (XDR; i.e. susceptible to only one or two classes of antibiotics)/ pan-drug-resistant (PDR; i.e. not susceptible to any antibiotics) nonfermenting Gram-negative bacteria and carbapenem-resistant Enterobacteriaceae (CRE) isolates. (Weak recommendation, low quality of evidence.)

Remarks: The panel finds it reasonable to consider selected patients at low risk for MDR pathogens (see Question 2) and some patients at high risk for MDR pathogens for initial empiric monotherapy, if there is a single-antibiotic therapy that is effective against $>90 \%$ of Gram-negative bacteria according to the local antibiogram. However, other clinical conditions, particularly severe illness or septic shock, may make individuals unsuitable for this recommendation.

\section{Benefits and harms}

Most published observational data suggest that survival of ICU patients with severe bacterial infections, including HAP/VAP, depends on early initiation of effective antimicrobial treatment, i.e. the aetiological 
microbe is sensitive to the therapeutic agent, and the dose administration route and infusion duration are optimal $[60,61]$. However, several studies in critically ill patients also suggest that combination broad-spectrum therapy may be associated with greater toxicity, and is a risk factor for the later emergence of multiresistant organisms and increased rates of superinfection [16, 58, 62-64].

We identified one systematic review and one meta-analysis which included 11 RCTs comparing combination with monotherapy therapy for the empirical treatment of VAP $[65,66]$. Of the 1805 patients enrolled in these trials, $85 \%$ were ventilated and 14\% were infected with Pseudomonas spp. Only two studies included late-onset VAP, and most excluded patients with septic shock and/or those with severe disease, as assessed by APACHE II (Acute Physiology and Chronic Health Evaluation II) score or other scoring systems. Monotherapy consisted of a broad-spectrum $\beta$-lactam with antipseudomonal activity in all studies except one, in which levofloxacin was used. Combination therapy consisted of a regimen combining a $\beta$-lactam and a fluoroquinolone (two RCTs) or an aminoglycoside (nine RCTs). Rates of mortality and treatment failure for combination therapy compared with monotherapy were similar in the two treatment options (eight RCTs). These results did not change in the sensitivity analyses of high-quality trials, trials enrolling only ventilated patients and trials including patients with clinically suspected pneumonia or microbiologically proven pneumonia. There were no significant differences in rates of superinfection or serious adverse events. Similar results were obtained in four RCTs comparing dual-versus single-antibiotic therapy for HAP/VAP (profiles 5 and 6 in the supplementary material) [67-70].

Interestingly, these results corroborate those of other RCTs conducted in ICU patients with severe sepsis or bloodstream infection [71], and other meta-analyses that have compared dual-antibiotic therapy versus monotherapy in settings other than the ICU and in different subsets of patients with other types of infection [72].

However, a substantial fraction of HAP/VAP episodes are associated with septic shock or severe disease, or are now caused by MDR Gram-negative bacteria, including XDR and PDR bacteria [18, 73, 74]. In these patients, a regimen initially combining two antibiotics targeting Gram-negative bacteria may increase the proportion of appropriately treated patients and may increase the rate of bacterial killing. Indeed, several observational studies have shown that the use of a regimen which initially combines a broad-spectrum $\beta$-lactam with an aminoglycoside increases the proportion of patients appropriately treated compared with monotherapy or to a regimen combining a $\beta$-lactam with a fluoroquinolone, particularly when the infection is due to MDR Gram-negative bacteria, such as ESBL-producing Enterobacteriaceae, $P$. aeruginosa or Acinetobacter spp. [75-79].

In addition, a systematic review and meta-analysis of randomised and observational studies concluded that combination antibiotic therapy decreased deaths related to high-risk life-threatening infections (particularly those associated with septic shock, including those with HAP/VAP) [80, 81]. Data were only calculated for monotherapy with a $\beta$-lactam and/or fluoroquinolone (as the primary treatment) and for combination treatment with these same primary agents. While the pooled analysis did not show differences in infection-related mortality between combination and monotherapy, the individual study estimates varied widely. In patients with shock/critical illness, combination therapy was associated with a significantly lower risk of death compared with monotherapy (12 studies; OR 0.51) (profile 7 in the supplementary material). A reduction in 28-day mortality was also seen in a recent retrospective cohort study in 4662 cases of culture-positive bacterial infection with septic shock treated with appropriate combination versus appropriate monotherapy (one study, 2446 patients; hazard ratio 0.77) [81]. The beneficial impact of combination therapy was restricted to patients treated with $\beta$-lactams in combination with aminoglycosides, fluoroquinolones or macrolides/clindamycin.

Available RCTs comparing dual- and single-antibiotic therapy in patients with VAP/HAP have specifically excluded patients with high disease severity. However, observational data suggest that combination antibiotic therapy (mainly with a broad-spectrum $\beta$-lactam combined with an aminoglycoside) may reduce mortality comparison with single therapy. The guideline panel considered that the reduction in mortality and other potential benefits outweigh the potential harms of combination antibiotic therapy in this subset of patients.

The first consideration in choosing an empiric therapy is whether the patient is at a high or low risk for both MDR pathogen infection and mortality. Low risk for mortality is defined as a $\leqslant 15 \%$ chance of dying, a mortality rate that has been associated with better outcome using monotherapy than combination therapy when treating serious infection (figure 2) [80]. For those at low risk, the recommended empiric therapy is a narrow-spectrum agent with activity against nonresistant Gram-negatives and methicillin-sensitive S. aureus (MSSA). Recommended choices are ertapenem, ceftriaxone, cefotaxime, moxifloxacin or levofloxacin. The use of third-generation cephalosporins increases the risk of $C$. difficile infections and MDR spread compared with penicillins or quinolones. 




FIGURE 2 Empiric antibiotic treatment algorithm for hospital-acquired pneumonia (HAP)/ventilator-associated pneumonia (VAP). MDR: multidrug-resistant; ICU: intensive care unit; MRSA: methicillin-resistant Staphylococcus aureus. \#: low risk for mortality is defined as a $\leqslant 15 \%$ chance of dying, a mortality rate that has been associated with better outcome using monotherapy than combination therapy when treating serious infection [80].

For the high-risk population, at risk for MDR pathogens, initial empiric therapy is determined by whether the patient is in septic shock or not. For those who are not in septic shock and who are treated in an ICU where a single broad-spectrum agent is active against $>90 \%$ of the likely Gram-negative pathogens, based on a local antibiogram, a single agent can be used against Gram-negatives. This should be chosen from agents that are active against $P$. aeruginosa, including imipenem, meropenem, cefepime, piperacillin/ tazobactam, levofloxacin or ceftazidime. If there is no risk for MRSA present in these patients, then any of the monotherapy agents that are active against Pseudomonas would be an effective choice, with the exception of aztreonam, which has no Gram-positive activity and should have another agent added to cover MSSA.

For high-risk patients who are not in septic shock, but who are treated in an ICU where $>25 \%$ of the $S$. aureus respiratory isolates in their ICU are MRSA, an agent with coverage for this pathogen should be added to initial empiric therapy. This involves choosing between vancomycin and linezolid.

For the high-risk patient, who is severely ill or in septic shock, initial empiric therapy should be with a dual-pseudomonal regimen plus MRSA coverage, the latter if the ICU has $>25 \%$ of $S$. aureus respiratory isolates as MRSA. The dual-pseudomonal regimen should also be chosen to provide coverage for Acinetobacter spp. and ESBL-producing Enterobacteriaceae, if these pathogens are prevalent in the ICU where the patient is being treated. The Gram-negative regimen should include an antipseudomonal $\beta$-lactam plus a second agent such as an aminoglycoside or an antipseudomonal quinolone (ciprofloxacin or levofloxacin). However, in some ICUs, particularly if Acinetobacter is a possible pathogen, the second agent will need to be colistin. The antipseudomonal $\beta$-lactams include imipenem, meropenem, cefepime, piperacillin/tazobactam, ceftazidime and aztreonam. If an aminoglycoside is added (an agent that adds additional Gram-negative coverage), it should be chosen from gentamicin, tobramycin and amikacin, but in many ICUs amikacin is the most active agent in this setting. For ESBL-producing organisms, a third-generation cephalosporin is not reliable and preferred therapy is with a carbapenem, but there may be some role for cefepime and piperacillin/tazobactam depending on local susceptibilities.

\section{Continuation of antibiotic therapy}

Clinical trials and observational studies have assessed initial empiric administration of single-antibiotic treatment or combination of antibiotics, but few have addressed the continuation of combination therapy. The two most relevant reasons for prescribing combined antibiotics for the entire treatment duration are to enhance treatment efficacy and prevent resistant strain emergence. However, such regimens did not prevent antimicrobial resistance emergence during therapy and were associated with significantly higher nephrotoxicity [72].

In most situations treatment can be safely switched to monotherapy after 3-5 days, provided that the initial therapy was appropriate, the clinical evolution is favourable and microbiological data do not 
indicate the presence of very-difficult-to-treat microorganisms, such as XDR/PDR Gram-negative bacteria and CRE. For these latter situations, multiple observational studies have reported lower mortality with combination antimicrobial therapy than with monotherapy [82-84]. The outcomes appear to be especially favourable when patients receive treatment with a carbapenem and a second agent, such as colistin, tigecycline and gentamicin, for the duration of treatment, but the best approach is yet to be defined [83, 84].

The guideline panel considered that continuing a regimen combining two effective antibiotics for the entire duration of treatment of therapy probably has more undesirable than beneficial effects, except in patients with infection caused by an XDR/PDR pathogen.

Relative importance of the outcomes

The panel valued above all the benefits in mortality, and placed equal value on the avoidance of drug adverse events and emerging antibiotic resistance

\section{Resource use}

No appropriate cost-effectiveness studies were identified. The panel took into consideration the reduced direct costs related to avoiding the overuse of dual broad-spectrum antibiotics, and the potentially high costs related to the anticipated drug adverse events and the emergence of antibiotic resistance with the routine prolonged use of dual broad-spectrum antibiotics.

\section{Question 4: In patients with HAP/VAP can duration of antimicrobial therapy be shortened to 7-10 days for certain populations, compared with 14 days, without increasing rates of relapsing infections or decreasing clinical cure? \\ Recommendations}

We suggest using a 7-8-day course of antibiotic therapy in patients with VAP without immunodeficiency, cystic fibrosis, empyema, lung abscess, cavitation or necrotising pneumonia and with a good clinical response to therapy. (Weak recommendation, moderate quality of evidence.)

Remarks: This recommendation also includes patients with nonfermenting Gram-negatives, Acinetobacter spp. and MRSA with a good clinical response. Longer courses of antibiotics may be needed in patients with inappropriate initial empiric therapy, and should be individualised to the patient's clinical response, specific bacteriological findings (such as PDR pathogens, MRSA or bacteraemia) and the serial measurement of biomarkers when indicated (see Question 6 and table 3).

The panel believes that applying the rationale and recommendations used for VAP in nonventilated patients with HAP represents good practice. (Good practice statement.)

We suggest against routine treatment with antibiotics for $>3$ days in patients with low probability of HAP and no clinical deterioration within $72 \mathrm{~h}$ of symptom onset. (Weak recommendation, low quality of evidence.)

Remarks: The term "low probability of HAP" refers to patients with low Clinical Pulmonary Infection Score (CPIS) scores or a clinical presentation not highly suggestive of pneumonia (e.g. $\leqslant 6)$ at symptom onset and continuing up to $72 \mathrm{~h}$.

\section{Benefits and harms}

We identified two recent systematic reviews which included six RCTs comparing short (7-8 days) with long (10-15 days) durations of antibiotic therapy in mixed early- and late-onset VAP populations [85, 86]. Two French studies compared 8-15-day antibiotic regimens, two others compared 7-10-day antibiotic regimens and one study compared 8-12-day antibiotic regimens [87-91]. One study [92] discontinued the antibiotic at day 3 if the CPIS score was $<7$, while others continued the treatment at the discretion of the treating physician. Most studies excluded patients with immunosuppression, cystic fibrosis and patients with lung abscess or empyema. Immunosuppression was defined as leukocytes $<1000 \mu \mathrm{L}^{-1}$, neutrophils $<500 \mu \mathrm{L}^{-1}$, acquired or congenital immunodeficiency syndrome, or use of immunosuppressants or long-term corticosteroids $\left(\geqslant 0.5 \mathrm{mg} \cdot \mathrm{kg}^{-1} \cdot \mathrm{day}^{-1}\right)$. Although one study required the presence of organ failure and sepsis, the others included mainly patients with nonsevere disease.

There was no difference between short and long courses of antibiotics with regard to mortality (up to 28 days), duration of mechanical ventilation or length of ICU stay, nor were there differences in mortality in the subset of patients with nonfermenting Gram-negative bacteria, although the number of events was limited (profile 8 in the supplementary material).

There were no significant differences in relapse rate between short and long courses, although there was a strong trend toward lower relapse in the long-course treatment, clearly driven by data from CHASTRE et al. 
[87]. Most of the patients with relapse in that study had VAP due to nonfermenting Gram-negative bacteria; there were no differences in relapse rate in patients with VAP due to other pathogens.

Antibiotic-free days were significantly higher in the short-course treatment and the incidence of secondary infections, i.e. VAP, due to MDR bacteria [86] was lower with the short-course than with the long-course regimen ( $43 \%$ versus $58 \%$ ), although the difference was not statistically significant. However, this difference reached statistical significance when a study published only in abstract form [91] was considered together with Chastre et al.'s study [87]. Adverse events were reported differently across studies. Treatment discontinuation due to adverse events may be similar with the two treatment options and shorter treatment duration is expected to be associated with better tolerability (profile 8 in the supplementary material).

There is no evidence relating to patients with a high probability of HAP (who were not mechanically ventilated). However, one study found that in patients with possible HAP and a low CPIS score (low clinical suspicion), a 3-day course of antibiotic therapy was associated with a significantly lower risk of superinfection and emergence of antimicrobial resistance than with a long therapy course (profile 9 in the supplementary material) [92].

\section{Relative importance of the outcomes}

The panel considered not only survival and avoidance of relapse as critical end-point variables, but also avoidance of individual (adverse events) and collective (emergence of antimicrobial resistance) collateral damage. All these factors were considered to be more critical than potential therapeutic failure.

\section{Resource use}

No appropriate cost-effectiveness studies were identified. The panel took into consideration the potentially high costs related to the future emergence of antibiotic resistance with the routine use of a prolonged course of antibiotics and the reduced direct costs related to a short course of antibiotics.

Balance between desirable effects and undesirable effects

The trade-off between beneficial and undesirable effects favoured short courses of antibiotics in immunocompetent patients with early- or late-onset VAP, without cystic fibrosis, empyema, lung abscess, cavitation or necrotising pneumonia. In patients with VAP due to nonfermenting Gram-negative bacteria, a routine 14-day course of antibiotics probably has more undesirable than beneficial effects, at least in patients with signs of quick response to treatment.

Any VAP or HAP in patients with cavitation or abscess formation, or with necrotising radiological characteristics, MRSA pneumonia, secondary bacteraemia or concomitant endocarditis, should be excluded from short-course therapy.

Question 5: In patients receiving antibiotic treatment for VAP or HAP, is bedside clinical assessment equivalent to the detection of serial biomarkers to predict adverse outcomes/clinical response at 72-96 $h$ ?

Recommendations

The panel believes that performing routine bedside clinical assessment in patients receiving antibiotic treatment for VAP or HAP represents good practice. (Good practice statement.)

Remarks: Clinical evaluation usually involves measurement of temperature, tracheobronchial secretion volume, culture and purulence assessment of tracheobronchial secretions, evaluation for chest radiograph resolution, white blood cell count, arterial oxygen tension/inspiratory oxygen fraction ratio $\left(\mathrm{PaO}_{2} / \mathrm{FIO}_{2}\right)$, and calculation of one or more scores such as CPIS, ODIN (Organ Dysfunction and Infection System), SOFA (Sequential Organ Failure Assessment), SAPS II (Simplified Acute Physiological Score II) and APACHE II.

We do not recommend routinely performing biomarker determinations in addition to bedside clinical assessment in patients receiving antibiotic treatment for VAP or HAP to predict adverse outcomes and clinical response at 72-96 h. (Strong recommendation, moderate quality of evidence.)

Remarks: Biomarker determinations may include C-reactive protein (CRP), procalcitonin (PCT), copeptin and mid-regional pro-atrial natriuretic peptide (MR-proANP). Clinicians should take into consideration the availability, feasibility and costs of each biomarker before routine testing.

\section{Benefits and harms}

There are no RCTs assessing treatment outcomes of patients with HAP/VAP managed according to clinical evaluation or according to serial biomarker measurements. 
Clinical evaluation includes bedside assessment, checking for improvement or worsening of temperature, tracheobronchial secretion volume, culture and purulence, chest radiograph, white blood cell count, $\mathrm{PaO}_{2} /$ $\mathrm{FIO}_{2}$ ratio, and CPIS [93], ODIN, SOFA and APACHE II scores. Some studies have demonstrated a benefit of serial CPIS assessments in predicting outcomes as early as day $3[94,95]$. With measurement of SOFA [96-98], SAPS II and APACHE II scores at the onset of VAP and serially, prognosis can be predicted; increasing SOFA and lack of improvement of the $\mathrm{PaO}_{2} / \mathrm{FIO}_{2}$ ratio [98] during the days following the onset of VAP have been associated with nonsurvival (profiles 10 and 11 in the supplementary material).

\section{Biomarkers}

Several prospective observational studies were conducted to assess the value of serum biomarker concentration and kinetics for predicting the outcome in HAP and/or VAP, including CRP, PCT, copeptin and MR-proANP.

CRP

Three studies in HAP/VAP patients assessed serial CRP measured at least three times a week after antibiotic prescription. In one study using a time-dependent analysis of the relative CRP concentration, CRP levels were significantly higher as early as day 4 in VAP patients with poor outcome than in those with a good outcome. In addition, the authors described different patterns of CRP response to antibiotics which were useful to predict the individual clinical course [99]. In another study, CRP concentrations fell between days 1 and 7 in all VAP patients, but were significantly higher in patients with unfavourable outcomes [95]. In the third study, CRP was measured in patients with HAP and VAP, and CRP ratios were calculated from HAP/VAP onset until day 10. Patients were classified according to the CRP ratios as "good" responders (CRP ratios $<0.67$ at day 10) or "poor" responders (nonresponse or biphasic response) [100]. Mortality rates were $53 \%$ in the poor response group $(n=34)$ and $20 \%$ in the good response group $(\mathrm{n}=30)(\mathrm{p}=0.01)$.

\section{PCT}

Some studies investigated the value of PCT as a prognostic marker during VAP. One study demonstrated that PCT was elevated at the onset of VAP in nonsurvivors [95]. In studies of PCT kinetics, although the levels of this biomarker decreased during the clinical course of all VAPs, they were significantly higher on days 1,3 and 7 in patients with an unfavourable outcome, and predicted a poor outcome in the multivariate analysis [95]. One observational study of 45 patients who developed VAP assessed their PCT and CRP levels from the day of VAP diagnosis to day 7 [100]. The authors found that PCT levels at days 3 and 7 were significantly higher in nonsurvivors than in survivors, and that PCT levels at day 3 were the strongest predictor of mortality. PCT levels fell significantly from day 0 to day 7 in the survivor group, but CRP levels did not.

\section{Copetin}

Two prospective studies showed that copeptin levels at VAP onset were significantly elevated in nonsurvivors and were lower in eventual survivors than in eventual nonsurvivors [96, 97].

\section{MR-proANP}

In one study, on day 0, MR-proANP concentration had the highest positive likelihood ratio (2.71) as a predictor of outcome in VAP [97]. In the study by BoEcK et al. [96], MR-proANP was higher at the onset of VAP in nonsurvivors than in survivors and in a logistic regression model it was identified as the best predictor of survival.

\section{Comparison of clinical and biomarker evaluations}

Two studies compared CRP and CRP ratios with clinical parameters in different groups of HAP/ VAP-treated patients. In the study by Moreno et al. [101], at day 0 , the poor responders had significantly higher SOFA and APACHE II scores, and lower $\mathrm{PaO}_{2} / \mathrm{FIO}_{2}$ ratio, than those with good response, while other clinical parameters did not differ, and CRP ratios that showed significant differences between the two groups were found later, at day 4. POVOA et al. [99] studied 47 patients with bacteriologically confirmed VAP, at day 0, CRP. Body temperature and white blood cell count of survivors and nonsurvivors did not differ, while in the multivariate analysis only day 4 CRP ratio ( 0.1 increase), age and day 0 SOFA score were independent predictors of death. Two other studies compared clinical assessment with PCT and CRP levels. In the first, multivariate analyses retained serum PCT levels and $P_{\mathrm{aO}_{2}} / F \mathrm{FIO}_{2}$ ratio on days 1, 3 and 7 as strong predictors of unfavourable outcome [95]. The other study was performed to determine the prognostic value of PCT and CRP kinetics in critically ill patients who developed VAP. In a multivariate logistic regression model, only decreasing $\triangle$ PCT (PCT day 4-PCT day 0) and decreasing $\triangle \mathrm{CRP}$ (CRP day 4-CRP day 0) were significantly lower in survivors [98]. 
The predictive value of copeptin and clinical assessment, including $\mathrm{PaO}_{2} / \mathrm{FIO}_{2}$ ratio and SOFA, ODIN and CPIS scores, were investigated [102]. The SOFA score and the copeptin levels at VAP onset were significantly elevated in nonsurvivors. The predictive value of serially measured SOFA significantly exceeded that of single SOFA and copeptin measurements. Regarding the effect of appropriate therapy, one study in patients with sepsis showed that PCT kinetics within the first days was associated with the appropriateness of antibiotic therapy and the overall survival [103], and another study in patients with VAP found that CRP was a surrogate of bacterial burden and that the follow-up measurement of CRP anticipates the appropriateness of antibiotic therapy [104].

There are some circumstances, such as renal impairment [105], haemodialysis [106], haemofiltration [107] and after resuscitated cardiac arrest [108], in which PCT has no value or the accepted thresholds have to be modified.

\section{Relative importance of the outcomes}

The panel valued above all the benefits in mortality, and secondarily the benefits in other unfavourable outcomes such as VAP recurrence or extrapulmonary infection requiring antibiotics before day 28, particularly in patients receiving adequate antimicrobial therapy.

\section{Resource use}

No appropriate cost-effectiveness studies have been identified comparing the use of bedside clinical assessment with the detection of serial biomarkers to predict adverse outcomes/clinical response at 72-96 h. The panel took into consideration the potentially high costs associated with the detection of serial biomarkers in relation to their limited prognosis capacity.

Question 6: In patients with HAP with severe sepsis or VAP, can serum PCT be used to reduce the duration of antibiotic therapy, compared with care that is not guided by serial biomarker measurements?

Recommendations

We do not recommend the routine measurement of serial serum PCT levels to reduce duration of the antibiotic course in patients with HAP or VAP when the anticipated duration is 7-8 days. (Strong recommendation, moderate quality of evidence.)

The panel believes that the measurement of serial serum PCT levels together with clinical assessment in specific clinical circumstances (table 3) with the aim of reducing antibiotic treatment duration represents good practice. (Good practice statement.)

\section{Benefits and harms}

Three RCTs including a total of 308 patients compared the discontinuation of antibiotic therapy according to serum PCT levels with the standard duration of antibiotics in patients with HAP or VAP as defined by clinical assessment [109-111]. More recently, a large RCT [112] in patients critically ill due to different causes provided data for HAP/VAP patients. In these studies, however, the standard duration of antibiotic therapy was substantially longer than 7-8 days; in one study, $>50 \%$ of the standard duration population were on antibiotics for $>14$ days [109], while in the most recent study the median duration of antibiotic treatment was 7 days [112]. In addition, for the most part these studies excluded patients with initially inappropriate antibiotic therapy and patients with severely compromised immunity.

Routine determination of serum PCT reduces the duration of antibiotic therapy by 3.2 days and is associated with a significant reduction in 28-day mortality (four RCTs, 748 patients; OR 0.67) but no differences in in-hospital mortality, although data on this latter outcome are limited [110]. The number of patients with failure of pneumonia resolution, overall recurrence, duration of ICU stay and duration of

TABLE 3 Patients in whom short duration of therapy may not be possible and in whom duration of therapy should be individualised

Initially inappropriate antibiotic therapy

Severely immunocompromised patients (such as neutropenia or stem cell transplant)

Highly antibiotic-resistant pathogens:

Pseudomonas aeruginosa

Carbapenem-resistant Acinetobacter spp.

Carbapenem-resistant Enterobacteriaceae

Second-line antibiotic therapy (e.g. colistin, tigecycline) 
mechanical ventilation were similar in the PCT-guided discontinuation strategy and standard antibiotic duration groups (profile 12 in the supplementary material). However, intentional short duration therapy (78 days) was not routinely used in the standard duration group. One systematic review [113] including five RCTs and two additional recent studies $[111,114]$ evaluated antibiotic discontinuation according to serum PCT levels in comparison with the standard antibiotic duration in critically ill patients with different pathologies. Pooled data showed similar trends, with a mean reduction of antibiotic duration of 2 days (five RCTs, 1.96 days). Mortality at 28 days was reduced in those patients managed according to PCT levels (four RCTs, 2347 patients; relative risk (RR) 0.84)), but no differences in hospital mortality were detected.

The guideline panel considered that an equivalent reduction in the antibiotic duration can be achieved by compliance with the 7-8-day treatment period suggested for patients with nosocomial pneumonia and without risk factors necessitating longer duration (table 3). In this population, the anticipated benefit of routine serum PCT measurement will be minimal or zero and will add costs in the majority of cases.

In patients with severely compromised immunity, inappropriate initial antibiotic therapy and infection with Pseudomonas and other nonfermenters, the evidence for the safety of routine 7-8-day antibiotic duration is less clear. Given the difficulties in assessing successful antibiotic treatment with clinical parameters or CPIS score alone [115], the guideline panel felt that serial PCT measurements may provide additional objective data to supplement clinical criteria in patients for whom the safety of 7-8 days of appropriate therapy has not been demonstrated (table 3). In all these circumstances shown in table 3 where short duration therapy is not well studied (just as there are exclusions for PCT studies and there are generally exclusions for short duration studies) we recommend an individualised duration of therapy, and serial PCT measurements are one factor to be considered. Since these patients are typically treated with longer courses of antibiotics, discontinuation of treatment based on PCT may result in cost savings and less selection for superinfection.

Initially inappropriate antibiotic therapy may result in a delay in clinical response [94, 113]. Serial PCT levels may therefore assist in planning the duration of therapy in these patients, but have not been validated to decide on antibiotic therapy initiation. The shortest duration of appropriate antibiotic treatment in patients with severely compromised immunity is unknown, as these patients were also excluded from RCTs of antibiotic duration for HAP/VAP and severe sepsis PCT trials.

The most problematic issue facing the attempts to shorten the duration of antibiotic courses is the higher recurrence rate recorded with 7-8 days than with 15 days of treatment, particularly for Pseudomonas and other nonfermenters [87]. However, 59\% of patients with VAP due to nonfermenters did not present recurrence after 8 days of treatment. Serial PCT may be valuable to identify these patients. By extension, treatment of other highly resistant bacteria causing HAP/VAP with suboptimal antibiotics, e.g. CRE or Acinetobacter spp. with second-line agents such as colistin or tigecycline, may be optimised by monitoring serial PCT levels.

Other biomarkers such as CRP may be useful, but are more prone to persistent elevation due to the noninfectious inflammatory disorders common in the ICU population. Availability of testing is the primary consideration for using an alternative to PCT.

\section{Relative importance of the outcomes}

The panel prioritised an easy-to-perform and inexpensive measure like the reduction of antibiotic duration in low-risk patients over a routine laboratory measurement, given the uncertainty of the benefits of laboratory values in patients who are already receiving a short course of antibiotic treatment.

\section{Resource use}

A cost-effectiveness study performed in Canada showed that 6 days of routine PCT measurement costs seemed to be balanced by a 2-day reduction in antibiotic treatment duration (i.e. the cost of testing equalled the cost of antibiotics saved) [111].

Table 3 summarises some of these selected groups where serial PCT measurement may be of some value. The evidence is only indirect, because to a large extent these patients have been excluded from clinical trials and thus the anticipated benefits of PCT detection are very uncertain.

Question 7: In patients requiring mechanical ventilation for $>48 h$, does topical application of nonabsorbable antimicrobials (antibiotics or chlorhexidine) in the oropharynx (SOD) or in the oropharynx and intestinal tract along with intravenous antibiotics (SDD) reduce the risk of VAP occurrence and/or improve patient outcome compared with standard care? (Standard care being treatment dispensed in ICU by the medical team in their usual manner)

Recommendations

The guideline panel decided not to issue a recommendation on the use of chlorhexidine to perform selective oral decontamination (SOD) in patients requiring mechanical ventilation until more safety data 
become available, due to the unclear balance between a potential reduction in pneumonia rate and a potential increase in mortality. (No formal recommendation.)

We suggest the use of SOD, but not SDD, in settings with low rates of antibiotic-resistant bacteria and low antibiotic consumption (where low antibiotic consumption in the ICU is $<1000$ daily doses per 1000 admission days). (Weak recommendation, low quality of evidence.)

Remarks: Although establishing a cut-off value for low and high resistance settings is a dilemma, the committee felt that a 5\% threshold was reasonable.

\section{Oral decontamination with chlorhexidine}

We identified several recent systematic reviews and one network meta-analysis that compared the use of oral chlorhexidine with usual care [110, 116-120].

In 16 RCTs including 3630 patients, the use of chlorhexidine was associated with a significant reduction of lower respiratory tract infections, including HAP and VAP (RR 0.73), but a nonsignificant increase in mortality (RR 1.13) [116]. There were no significant differences in mean duration of mechanical ventilation or ICU length of stay. Data on hospital length of stay and antibiotic prescriptions were limited. In patients undergoing cardiac surgery, chlorhexidine showed a similar effect, compared with usual care, in the reduction of lower respiratory tract infections including HAP and VAP (RR 0.56). Data about mortality were very limited and did not show a clear effect in this subgroup. Mean duration of mechanical ventilation or ICU length of stay were similar for the chlorhexidine and usual care groups. The analysis of noncardiac surgery studies showed that chlorhexidine was associated with a decreased prevalence of VAP (RR 0.78), but excess deaths (RR 1.13; 45 more per 1000 patients), although neither reached statistical significance (profile 13 in the supplementary material).

In 17 RCTs with a total of 2402 patients, excluding patients undergoing cardiac surgery due to short-term duration of mechanical ventilation, chlorhexidine was associated with a significant reduction in VAP incidence and a nonsignificant increase in mortality [117]. Similarly, there was no evidence that duration of mechanical ventilation or duration of ICU stay had any impact on outcomes.

Benefits and harms

The effects attributed to chlorhexidine were similar to those described in previous systematic reviews, although none of these included the same studies due to slight differences in the inclusion and exclusion criteria [118-120]. The association of chlorhexidine use with possible excess mortality was also seen in a recent network meta-analysis [121]. One explanation for this may be lung injury associated with aspiration of small amounts of chlorhexidine, but no clear relationship with dose and regimen can be established [122].

Possible explanations for the inconsistency of the effects observed in these studies may include differences in patient populations and differences in the outcomes assessed. The duration of mechanical ventilation in cardiac surgery patients is typically shorter than in noncardiac surgery patients. Studies of cardiac surgery patients typically assessed lower respiratory tract infection, not just VAP, and were excluded from the Cochrane systematic review [117]. Preventing VAP in persistently intubated patients is probably more difficult than preventing post-operative infections in extubated patients.

The absence of a clear pay-off between clinical benefits and the potential increase in mortality associated with chlorhexidine, and uncertainties regarding the appropriate dose, regimens and formulations, prevented the guideline panel from developing recommendations until further evidence becomes available about its effectiveness.

Relative importance of the outcomes

There was a wide discrepancy in the panel's views regarding the benefits of chlorhexidine in reducing nosocomial pneumonia and the potential risks associated with its use. As a result, no recommendation could be made.

Resource use

Although we did not identify any cost-effectiveness analyses for chlorhexidine use, any increase in mortality risk would have a heavy impact on healthcare costs.

Selective oropharyngeal decontamination and selective digestive decontamination

To reduce the incidence of infectious complications in patients requiring mechanical ventilation, two main antibiotic prophylaxis approaches have been proposed: SDD and SOD. SDD consists of oropharyngeal 
(applied as a paste) and gastric administration (through a nasogastric tube) of nonabsorbable antibiotics along with i.v. antibiotics. In SOD, the topical antibiotic paste is applied to the oropharynx alone.

We identified several recent systematic reviews and one network meta-analysis that assessed the effectiveness of either SOD or SDD [118-120, 123, 124].

\section{Selective oropharyngeal decontamination}

Three RCTs with a limited sample size (281 patients) compared the use of SOD with topical nonabsorbable antibiotics to usual care [118]. These studies showed a marked reduction in the incidence of VAP (RR 0.27) and no significant differences in mortality, duration of mechanical ventilation or duration of ICU stay.

In contrast, SOD was associated with a significant reduction in mortality in four RCTs (4266 patients) in comparison to usual care and in the one network meta-analysis (OR 0.85) [121]. This systematic review includes the results of a large cluster randomised trial (profile 14 in the supplementary material) [121].

\section{Selective digestive decontamination}

The effectiveness of SDD with topical nonabsorbable antibiotics applied in the oropharyngeal and digestive tracts, along with i.v. antibiotics, was assessed in 17 RCTs and 4045 patients [125]. SDD with topical and systemic antibiotics was associated with a significant reduction in mortality (RR 0.75), even though some of the clinical trials included patients not receiving mechanical ventilation.

The reduction in mortality was confirmed in a network meta-analysis of 15 RCTs (7839 patients) in comparison to usual care (OR 0.73) [110]. This systematic review includes the results of a large cluster randomised trial [126]. The effect of SOD, SDD or usual care on mortality and antibiotic resistance was assessed in a large cluster randomised crossover trial in the Netherlands [126]. The study included 5939 patients with an expected duration of intubation of $>48 \mathrm{~h}$ or an expected ICU stay of $>72 \mathrm{~h}$. SDD consisted of i.v. cefotaxime and topical application of tobramycin, colistin and amphotericin B in the oropharynx and stomach. SOD consisted of oropharyngeal application only of the same antibiotics. The adjusted odds ratio of 28-day mortality was 0.83 for SDD (35 deaths less per 1000 patients treated) and 0.86 for SOD (29 deaths less per 1000 patients treated), with usual care being the reference group. Both interventions also reduced the number of patients with at least one episode of ICU bacteraemia or candidaemia, mainly due to $S$. aureus and glucose-nonfermenting Gram-negative species (profile 15 in the supplementary material) [126].

\section{Emergence of resistance: SOD and SDD}

The emergence of antimicrobial resistance has been assessed in both randomised and observational studies of SDD and SOD in ICUs [124]. In the study by DANEMAN et al. [124], compared with control groups (which received no intervention), SDD or SOD were not associated with a significant difference in the prevalence of colonisation or infection with Gram-positive antimicrobial-resistant pathogens, including MRSA (OR 1.46) or vancomycin-resistant enterococci (OR 0.63).

Prevalence of Gram-negative bacteria resistant to selected antibiotics showed a trend toward a reduction for different antibiotics tested with the use of SDD. The reduction in resistant bacilli was significant for polymyxin E or B and third-generation cephalosporins. The study included randomised clinical trials in addition to large cluster randomised trials and nonrandomised trials, and so a selection bias cannot be excluded (profile 15 in the supplementary material) [124].

Recent metagenomic approaches and a recent trial comparing SDD with SOD reported an increase in the number of antibiotic resistance genes, especially of genes conferring resistance to aminoglycosides, in the gut flora from patients receiving SDD [127]. However, it has not yet been established whether this is directly related to the use of SDD or SOD.

In an earlier analysis, OosTDIJK et al. [128] looked at the ecological impact of SDD on all patients in their ICUs, rather than simply the resistance rates in patients randomised to SDD or SOD. This clinical trial was a cluster randomised crossover study with each unit using SDD, SOD or standard care for 6 months (with a 1-month wash-out/wash-in period between) in random order. The critically important aspect of this study is that the authors obtained prevalence of colonisation cultures from all patients in the ICU, not just those enrolled in the clinical trial. Approximately $70 \%$ of patients admitted during the study periods were expected to stay in the ICU for $<48 \mathrm{~h}$ and were therefore excluded. These patients represent a previously unstudied population whose colonisation patterns may be affected by SDD. A recent retraction of the data of this meta-analysis [129] reported changes in secondary outcomes in favour of SDD compared with SOD: lower mortality, reduced length of stay, lower rates of ICU-acquired bacteraemia and candidaemia, and lower prevalence of rectal carriage of antibiotic Gram-negative bacteria, but a more 
pronounced gradual increase in aminoglycoside-resistant Gram-negative bacteria. To look at rectal colonisation, OosTDIJK et al. [130] combined SOD and standard care periods, and found that its prevalence with ceftazidime-resistant bacteria significantly increased from 5-6\% before and during SDD to 15\% after the SDD period. Enterobacter spp. appeared to exert the greatest selection pressure. Prevalence of respiratory tract colonisation with ceftazidime-resistant isolates also gradually increased during SOD and SDD. In another study, the faecal and gut flora was importantly modified or significantly suppressed (Enterobacteriaceae) as a consequence of SDD compared with SOD or to standard of care [131]. In a follow-up study, DE SMET et al. [132] found trends for higher rates of hospital-acquired infections in patients discharged from ICUs that had received either SDD or SOD.

\section{Benefits and harms}

Most of the studies were performed in countries and settings with low levels of antibiotic resistance and the study conclusions are primarily applicable to these contexts. Effectiveness of SOD or SDD in settings with high levels of antibiotic resistance has not been assessed.

In settings with low levels of antibiotic resistance, SOD (with topical nonabsorbable antibiotics) and SDD (with oropharyngeal and digestive tube administration of topical nonabsorbable antibiotics and i.v. antibiotics) may be associated with reductions in nosocomial pneumonia and death. The potential effects of antibiotic use on antimicrobial resistance are uncertain. Considering the clinical benefits of these two strategies to be similar, the guideline panel advocated the use of SOD and avoiding supplementary i.v. antibiotics as in SDD. It should be stressed that all these studies were performed at a time when VAP bundles were not routinely applied, and the incremental benefit of SOD and SDD to a VAP bundle is largely unknown.

The guideline panel recognised that establishing a cut-off value for low and high resistance settings is a dilemma, but a $5 \%$ threshold seems reasonable.

\section{Relative importance of the outcomes}

The panel placed similar value on the prevention of mortality, nosocomial pneumonia and the emergence of resistance.

\section{Resource use}

A recent cost-effectiveness analysis showed that both SOD and SDD are cost-saving and are more effective than usual care, based on a post hoc analysis of 5920 patients from a crossover study using cluster randomisation in 13 ICUs, all in the Netherlands [133].

\section{Summary}

In these international guidelines, a panel of experts appointed by the ERS, ESICM, ESCMID and ALAT provide recommendations for seven PICO questions regarding diagnosis, empirical and definitive antibiotic therapy, and prevention of HAP and VAP following a GRADE approach (table 1).

\section{Acknowledgements}

We want to thank Thomy Tonia (ERS methodologist; University of Bern, Bern, Switzerland) for her valuable methodological advice, and Elisabeth Sancho (CIBERES, Barcelona, Spain) for her administrative and secretarial support.

\section{References}

1 American Thoracic Society, Infectious Diseases Society of America. Guidelines for the management of adults with hospital-acquired, ventilator-associated, and healthcare-associated pneumonia. Am J Respir Crit Care Med 2005; 171: 388-416.

2 Craven DE, Hudcova J, Lei Y. Diagnosis of ventilator-associated respiratory infections (VARI): microbiologic clues for tracheobronchitis (VAT) and pneumonia (VAP). Clin Chest Med 2011; 32: 547-557.

3 Rosenthal VD, Bijie H, Maki DG, et al. International Nosocomial Infection Control Consortium (INICC) report, data summary of 36 countries, for 2004-2009. Am J Infect Control 2012; 40: 396-407.

4 Rosenthal VD, Maki DG, Jamulitrat S, et al. International Nosocomial Infection Control Consortium (INICC) report, data summary for 2003-2008, issued June 2009. Am J Infect Control 2010; 38: 95-104.

5 Cook DJ, Walter SD, Cook RJ, et al. Incidence of and risk factors for ventilator-associated pneumonia in critically ill patients. Ann Intern Med 1998; 129: 433-440.

6 Rello J, Ollendorf DA, Oster G, et al. Epidemiology and outcomes of ventilator-associated pneumonia in a large US database. Chest 2002; 122: 2115-2121.

7 Asehnoune K, Seguin P, Allary J, et al. Hydrocortisone and fludrocortisone for prevention of hospital-acquired pneumonia in patients with severe brain injury (Corti-TC): a double blind multicenter phase 3, randomised placebo-controlled trial. Lancet Respir Med 2014; 2: 706-716.

8 Warren DK, Shukla SJ, Olsen MA, et al. Outcome and attributable cost of ventilator-associated pneumonia among intensive care unit patients in a suburban medical center. Crit Care Med 2003; 31: 1312-1317. 
Stone PW, Braccia D, Larson L. Systematic review of economic analyses of health care-associated infections. Am $J$ Infect Control 2005; 33: 501-509.

10 Wyncoll D, Camporata L. Number needed to treat and cost-effectiveness in the prevention of ventilator-associated pneumonia. Crit Care 2012; 16: 430.

11 Erbay RH, Yalcin AN, Zencir M, et al. Costs and risks factors for ventilator-associated pneumonia in a Turkish University Hospital's Intensive Care Unit: a case-control study. BMC Pulm Med 2004; 4: 1-7.

12 Falcone M, Venditti M, Shindo Y, et al. Healthcare-associated pneumonia: diagnostic criteria and distinction from community-acquired pneumonia. Int J Infect Dis 2011; 15: 545-550.

13 Polverino E, Torres A, Menendez R, et al. Microbial aetiology of healthcare associated pneumonia in Spain: a prospective, multicentre, case-control study. Thorax 2013; 68: 1007-1014

14 Safdar N, Crnich CJ, Maki DG. The pathogenesis of ventilator-associated pneumonia: its relevance to developing effective strategies for prevention. Respir Care 2005; 50: 725-739.

15 Langer M, Cigada M, Mandelli M, et al. Early onset pneumonia: a multicenter study in intensive care units. Intensive Care Med 1987; 13: 342-346.

16 Trouillet JL, Chastre J, Vuagnat A, et al. Ventilator-associated pneumonia caused by potentially drug-resistant bacteria. Am J Respir Crit Care Med 1998; 157: 531-539.

17 Martin-Loeches I, Torres A, Rinaudo M, et al. Resistance patterns and outcomes in intensive care unit (ICU)-acquired pneumonia. Validation of European Centre for Disease Prevention and Control (ECDC) and the Centers for Disease Control and Prevention (CDC) classification of multidrug resistant organisms. J Infect 2015; 74: 213-222.

18 Martin-Loeches I, Deja M, Koulenti D, et al. Potentially resistant microorganisms in intubated patients with hospital-acquired pneumonia: the interaction of ecology, shock and risk factors. Intensive Care Med 2013; 39: 672-681.

19 Ferrer M, Liapikou A, Valencia M, et al. Validation of the American Thoracic Society-Infectious Diseases Society of America guidelines for hospital-acquired pneumonia in the intensive care unit. Clin Infect Dis 2010; 50: 945-952.

20 Restrepo MI, Peterson J, Fernandez JF, et al. Comparison of the bacterial etiology of early-onset and late-onset ventilator-associated pneumonia in subjects enrolled in 2 large clinical studies. Respir Care 2013; 58: 1220-1225

21 Micek ST, Wunderink RG, Kollef MH, et al. An international multicenter retrospective study of Pseudomonas aeruginosa nosocomial pneumonia: impact of multidrug resistance. Crit Care 2015; 19: 219.

22 Garnacho-Montero J, Ortiz-Leyba C, Fernández-Hinojosa E, et al. Acinetobacter baumannii ventilator-associated pneumonia: epidemiological and clinical findings. Intensive Care Med 2005; 31: 649-655.

23 Melsen WG, Rovers MM, Bonten MJ. Ventilator-associated pneumonia and mortality: a systematic review of observational studies. Crit Care Med 2009; 37: 2709-2718.

24 Bekaert M, Timsit JF, Vansteelandt S, et al. Attributable mortality of ventilator-associated pneumonia: a reappraisal using causal analysis. Am J Respir Crit Care Med 2011; 184: 1133-1139.

25 Melsen WG, Rovers MM, Koeman M, et al. Estimating the attributable mortality of ventilator-associated pneumonia from randomized prevention studies. Crit Care Med 2011; 39: 2736-2742.

26 Nguile-Makao M, Zahar JR, Français A, et al. Attributable mortality of ventilator-associated pneumonia: respective impact of main characteristics at ICU admission and VAP onset using conditional logistic regression and multi-state models. Intensive Care Med 2010; 36: 781-789.

27 Torres A, Ewig S, Lode H. Defining, treating and preventing hospital acquired pneumonia: a European perspective. Intensive Care Med 2009; 35: 9-29.

28 Kalil AC, Metersky ML, Klompas M, et al. Management of adults with hospital-acquired and ventilator-associated pneumonia: 2016 clinical practice guidelines by the Infectious Diseases Society of America and the American Thoracic Society. Clin Infect Dis 2016; 63: e61-e111.

29 Klompas M, Kleinman K, Murphy MV. Descriptive epidemiology and attributable morbidity of ventilator-associated events. Infect Control Hosp Epidemiol 2014; 35: 502-510.

30 Bouadma L, Deslandes E, Lolom I, et al. Long-term impact of a multifaceted prevention program on ventilator-associated pneumonia in a medical intensive care unit. Clin Infect Dis 2010; 51: 1115-1122.

31 Tabah A, Cotta MO, Garnacho-Montero J, et al. A systematic review of the definitions, determinants, and clinical outcomes of antimicrobial de-escalation in the intensive care unit. Clin Infect Dis 2016; 62: 1009-1017.

32 Guyatt GH, Oxman AD, Vist GE, et al. An emerging consensus on rating quality of evidence and strength of recommendations. BMJ 2008; 336: 924-926.

33 Brożek JL, Akl EA, Compalati E, et al. Grading quality of evidence and strength of recommendations in clinical practice guidelines part 3 of 3. The GRADE approach to developing recommendations. Allergy 2011; 66: $588-595$.

34 Canadian Critical Care Trials Group. A randomized trial of diagnostic techniques for ventilator-associated pneumonia. N Engl J Med 2006; 355: 2619-2630.

35 Sole Violan J, Fernandez JA, Benitez AB, et al. Impact of quantitative invasive diagnostic techniques in the management and outcome of mechanically ventilated patients with suspected pneumonia. Crit Care Med 2000; 28: 2737-2741.

36 Berton DC, Kalil AC, Teixeira PJ. Quantitative versus qualitative cultures of respiratory secretions for clinical outcomes in patients with ventilator-associated pneumonia. Cochrane Database Syst Rev 2014; 10: CD006482.

37 Fagon JY, Chastre J, Wolff M, et al. Invasive and noninvasive strategies for management of suspected ventilator-associated pneumonia. A randomized trial. Ann Intern Med 2000; 132: 621-630.

38 Ewig S, Niederman M, Torres A. Management of suspected ventilator-associated pneumonia. Ann Intern Med 2000; 133: 1008-1009.

39 Bonten MJ, Bergmans DC, Stobberingh EE, et al. Implementation of bronchoscopic techniques in the diagnosis of ventilator-associated pneumonia to reduce antibiotic use. Am J Respir Crit Care Med 1997; 156: 1820-1824.

40 Leone M, Bourgoin A, Cambon S, et al. Empirical antimicrobial therapy of septic shock patients: adequacy and impact on the outcome. Crit Care Med 2003; 31: 462-467.

41 Timsit JF, Harbarth S, Carlet J. De-escalation as a potential way of reducing antibiotic use and antimicrobial resistance in ICU. Intensive Care Med 2014; 40: 1580-1582. 

ventilator-associated pneumonia and negative quantitative bronchoscopy cultures. Crit Care Med 2013; 41: $1656-1663$.

43 Kollef MH, Shorr A, Tabak YP, et al. Epidemiology and outcomes of health-care-associated-pneumonia: results from a large US database of culture positive pneumonia. Chest 2005; 128: 3854-3862.

44 Timsit JF. Bronchoalveolar lavage for VAP diagnosis: patients must be sampled before any change of antimicrobial therapy. Intensive Care Med 2007; 33: 1690-1693.

45 Dennesen PJ, van der Ven AJ, Kessels AG, et al. Resolution of infectious parameters after antimicrobial therapy in patients with ventilator-associated pneumonia. Am J Respir Crit Care Med 2001; 163: 1371-1375.

46 Prats E, Dorca J, Pujol M, et al. Effects of antibiotics on protected specimen brush sampling in ventilator-associated pneumonia. Eur Respir J 2002; 19: 944-951.

47 Souweine B, Veber B, Bedos JP, et al. Diagnostic accuracy of protected specimen brush and bronchoalveolar lavage in nosocomial pneumonia: impact of previous antimicrobial treatments. Crit Care Med 1998; 26: 236-244.

48 de Lassence A, Joly-Guillou ML, Salah A, et al. Accuracy of delayed (24 hours) processing of bronchoalveolar lavage for diagnosing bacterial pneumonia. Crit Care Med 2004; 32: 680-685.

49 Montravers P, Veber B, Auboyer C, et al. Diagnostic and therapeutic management of nosocomial pneumonia in surgical patients: results of the Eole study. Crit Care Med 2002; 30: 368-375.

50 Leroy O, Jaffré S, D’Escrivan T, et al. Hospital-acquired pneumonia: risk factors for antimicrobial-resistant causative pathogens in critically ill patients. Chest 2003; 123: 2034-2042.

51 Perbet S, Mongardon N, Dumas F, et al. Early-onset pneumonia after cardiac arrest: characteristics, risk factors and influence on prognosis. Am J Respir Crit Care Med 2011; 184: 1048-1054.

52 Arvanitis M, Anagnostou T, Kourkoumpetis TK, et al. The impact of antimicrobial resistance and aging in VAP outcomes: experience from a large tertiary care center. PLoS One 2014; 9: e89984.

53 Verhamme KM, De Coster W, De Roo L, et al. Pathogens in early-onset and late-onset intensive care unit-acquired pneumonia. Infect Control Hosp Epidemiol 2007; 28: 389-397.

54 Luna CM, Vujacich P, Niederman MS, et al. Impact of BAL data on the therapy and outcome of ventilator-associated pneumonia. Chest 1997; 111: 676-685.

55 Leone M, Garcin F, Bouvenot J, et al. Ventilator-associated pneumonia: breaking the vicious circle of antibiotic overuse. Crit Care Med 2007; 35: 379-385.

56 Shindo Y, Ito R, Kobayashi D, et al. Risk factors for drug-resistant pathogens in community-acquired and healthcare-associated pneumonia. Am J Respir Crit Care Med 2013; 188: 985-995.

57 Prina E, Ranzani OT, Polverino E, et al. Risk factors associated with potentially antibiotic-resistant pathogens in community-acquired pneumonia. Ann Am Thorac Soc 2015; 12: 153-160.

58 Kett $\mathrm{DH}$, Cano E, Quartin AA, et al. Implementation of guidelines for management of possible multidrug-resistant pneumonia in intensive care: an observational, multicentre cohort study. Lancet Infect Dis 2011; 11: 181-189.

59 Torres A, Lee N, Cilloniz C, et al. Laboratory diagnosis of pneumonia in the molecular age. Eur Respir J 2016; 48: 1764-1778.

60 Kumar A, Ellis P, Arabi Y, et al. Initiation of inappropriate antimicrobial therapy results in a fivefold reduction of survival in human septic shock. Chest 2009; 136: 1237-1248.

61 Kollef MH, Sherman G, Ward S, et al. Inadequate antimicrobial treatment of infections: a risk factor for hospital mortality among critically ill patients. Chest 1999; 115: 462-474.

62 Bliziotis IA, Samonis G, Vardakas KZ, et al. Effect of aminoglycoside and beta-lactam combination therapy versus beta-lactam monotherapy on the emergence of antimicrobial resistance: a meta-analysis of randomized, controlled trials. Clin Infect Dis 2005; 41: 149-158.

63 Koontz CS, Chang MC, Meredith JW. Effects of empiric antibiotic administration for suspected pneumonia on subsequent opportunistic pulmonary infections. Am Surg 2000; 66: 1110-1114.

64 Rello J, Ausina V, Ricart M, et al. Impact of previous antimicrobial therapy on the etiology and outcome of ventilator-associated pneumonia. Chest 1993; 104: 1230-1235.

65 Aarts MA, Hancock JN, Heyland D, et al. Empiric antibiotic therapy for suspected ventilator-associated pneumonia: a systematic review and meta-analysis of randomized trials. Crit Care Med 2008; 36: 108-117.

66 Heyland DK, Dodek P, Muscedere J, et al. Randomized trial of combination versus monotherapy for the empiric treatment of suspected ventilator-associated pneumonia. Crit Care Med 2008; 36: 737-744

67 Fernandez-Guerrero M, Gudiol F, Rodriguez-Torres A, et al. Nosocomial pneumonia: comparative multicentre trial between monotherapy with cefotaxime and treatment with antibiotic combinations. Infection 1991; 19: Suppl. 6, S320-S325.

68 Jaspers CA, Kieft H, Speelberg B, et al. Meropenem versus cefuroxime plus gentamicin for treatment of serious infections in elderly patients. Antimicrob Agents Chemother 1998; 42: 1233-1238.

69 Rubinstein E, Lode H, Grassi C. Ceftazidime monotherapy vs. ceftriaxone/tobramycin for serious hospital-acquired gram-negative infections. Antibiotic Study Group. Clin Infect Dis 1995; 20: 1217-1228.

70 Awad S, Rodríguez AH, Chuang Y, et al. A phase randomized double blind comparison of ceftobiprole medocaril versus ceftazidime plus linezolid for the treatment of hospital-acquired pneumonia. Clin Infect Dis 2014; 59: 51-61.

71 Brunkhorst FM, Oppert M, Marx G, et al. Effect of empirical treatment with moxifloxacin and meropenem vs meropenem on sepsis-related organ dysfunction in patients with severe sepsis: a randomized trial. JAMA 2012; 307: 2390-2399.

72 Paul M, Silbiger I, Grozinsky S, et al. Beta lactam antibiotic monotherapy versus beta lactam-aminoglycoside antibiotic combination therapy for sepsis. Cochrane Database Syst Rev 2006; 1: CD003344.

73 Brusselaers N, Labeau S, Vogelaers D, et al. Value of lower respiratory tract surveillance cultures to predict bacterial pathogens in ventilator-associated pneumonia: systematic review and diagnostic test accuracy meta-analysis. Intensive Care Med 2013; 39: 365-375.

74 Magiorakos AP, Srinivasan A, Carey RB, et al. Multidrug-resistant, extensively drug-resistant and pandrug-resistant bacteria: an international expert proposal for interim standard definitions for acquired resistance. Clin Microbiol Infect 2012; 18: 268-281. 
Chamot E, Boffi El Amari E, Rohner P, et al. Effectiveness of combination antimicrobial therapy for Pseudomonas aeruginosa bacteremia. Antimicrob Agents Chemother 2003; 47: 2756-2764.

Garnacho-Montero J, Sa-Borges M, Sole-Violan J, et al. Optimal management therapy for Pseudomonas aeruginosa ventilator-associated pneumonia: an observational, multicenter study comparing monotherapy with combination antibiotic therapy. Crit Care Med 2007; 35: 1888-1895.

Martinez JA, Cobos-Trigueros N, Soriano A, et al. Influence of empiric therapy with a beta-lactam alone or combined with an aminoglycoside on prognosis of bacteremia due to Gram-negative microorganisms. Antimicrob Agents Chemother 2010; 54: 3590-3596.

Micek ST, Reichley RM, Kollef MH. Health care-associated pneumonia (HCAP): empiric antibiotics targeting methicillin-resistant Staphylococcus aureus (MRSA) and Pseudomonas aeruginosa predict optimal outcome. Medicine 2011; 90: 390-395.

Micek ST, Welch EC, Khan J, et al. Empiric combination antibiotic therapy is associated with improved outcome against sepsis due to Gram-negative bacteria: a retrospective analysis. Antimicrob Agents Chemother 2010; 54: 1742-1748.

Kumar A, Safdar N, Kethireddy S, et al. A survival benefit of combination antibiotic therapy for serious infections associated with sepsis and septic shock is contingent only on the risk of death: a meta-analytic/ meta-regression study. Crit Care Med 2010; 38: 1651-1664.

Kumar A, Zarychanski R, Light B, et al. Early combination antibiotic therapy yields improved survival compared with monotherapy in septic shock: a propensity-matched analysis. Crit Care Med 2010; 38: 1773-1785.

Doi Y, Paterson DL. Carbapenemase-producing Enterobacteriaceae. Semin Respir Crit Care Med 2015; 36: 74-84.

Tumbarello M, Viale P, Viscoli C, et al. Predictors of mortality in bloodstream infections caused by Klebsiella pneumoniae carbapenemase-producing K. pneumoniae: importance of combination therapy. Clin Infect Dis 2012; 55: 943-950.

Tzouvelekis LS, Markogiannakis A, Piperaki E, et al. Treating infections caused by carbapenemase-producing Enterobacteriaceae. Clin Microbiol Infect 2014; 20: 862-872.

Dimopoulos G, Poulakou G, Pneumatikos IA, et al. Short- vs long-duration antibiotic regimens for ventilator-associated pneumonia: a systematic review and meta-analysis. Chest 2013; 144: 1759-1767.

Pugh R, Grant C, Cooke RPD, et al. Short-course versus prolonged-course antibiotic therapy for hospital-acquired pneumonia in critically ill adults. Cochrane Database Syst Rev 2015; 8: CD007577.

Chastre J, Wolff M, Fagon JY, et al. Comparison of 8 vs 15 days of antibiotic therapy for ventilator-associated pneumonia in adults: a randomized trial. JAMA 2003; 290: 2588-2598.

Capellier G, Mockly H, Charpentier C, et al. Early-onset ventilator-associated pneumonia in adults randomized clinical trial: comparison of 8 versus 15 days of antibiotic treatment. PLoS One 2012; 7: e41290.

Fekih Hassen M, Ayed S, Ben Sik Ali H, et al. Duree de l'antibiotherapie lors du traitement des pneumopathies acquises sous ventilation mecanique: comparaison entre sept jours et dix jours. Etude pilote. [Duration of antibiotic therapy for ventilator-associated pneumonia: comparison of 7 and 10 days. A pilot study.] Ann $\mathrm{Fr}$ Anesth Reanim 2009; 28: 16-23.

Kollef M, Chastre J, Restrepo M, et al. A randomized trial of 7-day doripenem versus 10-day imipenem-cilastatin for ventilator-associated pneumonia. Crit Care 2012; 16: R218.

Medina JC, Perez Protto SE, Paciel D, et al. Antibiotic treatment for the ventilator-associated pneumonia: 8 vs. 12 days randomized trial preliminary data. Annual Interscience Conference on Antimicrobial Agents and Chemotherapy, Chicago, IL, 2007.

Singh N, Rogers P, Atwood CW, et al. Short-course empiric antibiotic therapy for patients with pulmonary infiltrates in the intensive care unit. A proposed solution for indiscriminate antibiotic prescription. Am J Respir Crit Care Med 2000; 162: 505-511.

Pugin J, Auckenthaler R, Mili N, et al. Diagnosis of ventilator-associated pneumonia by bacteriologic analysis of bronchoscopic and nonbronchoscopic "blind" bronchoalveolar lavage fluid. Am Rev Respir Dis 1991; 143: $1121-1129$.

Luna CM, Blanzaco D, Niederman MS, et al. Resolution of ventilator-associated pneumonia: prospective evaluation of the clinical pulmonary infection score as an early clinical predictor of outcome. Crit Care Med 2003; 31: 676-682

Luyt CE, Guerin V, Combes A, et al. Procalcitonin kinetics as a prognostic marker of ventilator-associated pneumonia. Am J Respir Crit Care Med 2005; 171: 48-53.

Boeck L, Eggimann P, Smyrnios N, et al. Midregional pro-atrial natriuretic peptide and procalcitonin improve survival prediction in VAP. Eur Respir J 2011; 37: 595-603.

Seligman R, Seligman BGS, Teixeira PJ. Comparing the accuracy of predictors of mortality in ventilator-associated pneumonia. J Bras Pneumol 2011; 37: 495-503.

Seligman R, Meisner M, Lisboa TC, et al. Decreases in procalcitonin and C-reactive protein are strong predictors of survival in ventilator-associated pneumonia. Crit Care 2006; 10: R125.

Póvoa P, Coelho L, Almeida E, et al. C-reactive protein as a marker of ventilator-associated pneumonia resolution: a pilot study. Eur Respir J 2005; 25: 804-812.

Tanriverdi H, Tor MM, Kart L, et al. Prognostic value of serum procalcitonin and C-reactive protein levels in critically ill patients who developed ventilator-associated pneumonia. Ann Thorac Med 2015; 10: 137-142.

Moreno MS, Nietmann H, Matias CM, et al. C-reactive protein: a tool in the follow-up of nosocomial pneumonia. J Infect 2010; 61: 205-201.

Boeck L, Eggimann P, Smyrnios N, et al. The Sequential Organ Failure Assessment score and copeptin for predicting survival in ventilator-associated pneumonia. J Crit Care 2012; 27: 523.

Charles PE, Tinel C, Barbar S, et al. Procalcitonin kinetics within the first days of sepsis: relationship with the appropriateness of antibiotic therapy and the outcome. Crit Care 2009; 13: R38.

Lisboa T, Seligman R, Diaz E, et al. C-reactive protein correlates with bacterial load and appropriate antibiotic therapy in suspected ventilator-associated pneumonia. Crit Care Med 2008; 36: 166-171.

Amour J, Bienbaum A, Langeron O, et al. Influence of renal dysfunction on the accuracy of procalcitonin for the diagnosis of postoperative infection after vascular surgery. Crit Care Med 2008; 36: 1147-1154. 


$$
\text { patients }
$$
pneumonia. Cochrane Database Syst Rev 2013; 8: CD008367.

review and meta-analysis of randomized controlled trials. J Hosp Infect 2013; 84: 283-293.

119 Labeau SO, Van de Vyver K, Brusselaers N, et al. Prevention of ventilator-associated pneumonia with oral antiseptics: a systematic review and meta-analysis. Lancet Infect Dis 2011; 11: 845-854.

120 Pileggi C, Bianco A, Flotta D, et al. Prevention of ventilator-associated pneumonia, mortality and all intensive care unit acquired infections by topically applied antimicrobial or antiseptic agents: a meta-analysis of randomized controlled trials in intensive care units. Crit Care 2011; 15: R155.

121 Price R, MacLennan G, Glen J. Selective digestive or oropharyngeal decontamination and topical oropharyngeal chlorhexidine for prevention of death in general intensive care: systematic review and network meta-analysis. BMJ 2014; 348: g2197.

122 Xue Y, Zhang S, Yang Y, et al. Acute pulmonary toxic effects of chlorhexidine (CHX) following an intratracheal instillation in rats. Hum Exp Toxicol 2011; 30: 1795-1803.

123 Roquilly A, Marret E, Abraham E, et al. Pneumonia prevention to decrease mortality in intensive care unit: a systematic review and meta-analysis. Clin Infect Dis 2015; 60: 64-75.

124 Daneman N, Sarwar S, Fowler RA, et al. Effect of selective decontamination on antimicrobial resistance in intensive care units: a systematic review and meta-analysis. Lancet Infect Dis 2013; 13: 328-341.

125 D'Amico R, Pifferi S, Torri V, et al. Antibiotic prophylaxis to reduce respiratory tract infections and mortality in adults receiving intensive care. Cochrane Database Syst Rev 2009; 4: CD000022.

126 de Smet AMGA, Kluytmans JAJW, Cooper BS, et al. Decontamination of the digestive tract and oropharynx in ICU patients. N Engl J Med 2009; 360: 20-31.

127 Buelow E, Gonzalez TB, Versluis D, et al. Effects of selective digestive decontamination (SDD) on the gut resistome. J Antimicrob Chemother 2014; 69: 2215-2223.

128 Oostdijk EAN, Kesecioglu J, Schultz MJ, et al. Effects of decontamination of the oropharynx and intestinal tract on antibiotic resistance in ICUs: a randomized clinical trial. JAMA 2014; 312: 1429-1437.

129 Oostdijk EAN, Kesecioglu J, Schultz MJ, et al. Notice of retraction and replacement: effects of decontamination of the oropharynx and intestinal tract on antibiotic resistance in ICUs: a randomized clinical trial. JAMA 2017; 317: 1583-1584.

130 Oostdijk EAN, de Smet AMGA, Blok HE, et al. Ecological effects of selective decontamination on resistant Gram-negative bacterial colonization. Am J Respir Crit Care Med 2010; 181: 452-457.

131 Benus R, Harmsen H, Welling G, et al. Impact of digestive oropharyngeal decontamination on the intestinal microbiota in ICU patients. Intensive Care Med 2010; 36: 1394-1402.

132 de Smet AMGA, Hopmans TEM, Minderhoud ALC, et al. Decontamination of the digestive tract and oropharynx: hospital-acquired infections after discharge from Intensive care unit. Intensive Care Med 2009; 35: $160-1613$.

133 Branch-Elliman W, Wright SB, Howell MD. Determining the ideal strategy for ventilator-associated pneumonia prevention. Cost-benefit analysis. Am J Respir Crit Care Med 2015; 192: 57-63. 